Supporting Information for:

\title{
Characterization of Self-Assembled Monolayers of Porphyrins Bearing Multiple Thiol- Derivatized Rigid-Rod Tethers
}

\author{
Amir A. Yasseri, Dennis Syomin, Vladimir L. Malinovskii, Robert S. Loewe, \\ Jonathan S. Lindsey, Francisco Zaera, and David F. Bocian
}

Table of Contents:
A. Porphyrin Syntheses
$\mathrm{S} 1-\mathrm{S} 2$
B. Experimental Section
$\mathrm{S} 2-\mathrm{S} 4$
C. References
S5-S5
D. Schemes
S6-S9
E. Experimental Data
S10-S29

A. Porphyrin Syntheses. Compounds ZnPS $\mathbf{Z n}_{\mathbf{1}}$, cis-ZnPS $\mathbf{Z S}_{\mathbf{2}}$, trans-ZnPS $\mathbf{Z}_{\mathbf{2}}, \mathrm{ZnPS}_{\mathbf{3}}$, and $\mathbf{Z n P S}_{\mathbf{4}}$ described in the main text are hereby referred to as compounds S-5, S-12, S-15, S-6, and S-2, respectively.

The condensation of pyrrole and aldehyde $\mathbf{S - 1}{ }^{\mathrm{S} 1}$ was carried out using new cocatalysis conditions $\left[\mathrm{BF}_{3} \cdot \mathrm{O}(\mathrm{Et})_{2}-\mathrm{NaCl} \text { in } \mathrm{CH}_{2} \mathrm{Cl}_{2} \text { at room temperature }\right]^{\mathrm{S} 2}$ followed by oxidation with DDQ and metalation of the resulting free base porphyrin with zinc. In this manner, $\mathrm{A}_{4}$-porphyrin S-2 was prepared in 70\% yield after chromatographic workup (Scheme S-1). An homologous porphyrin bearing four $S$-acetylthio moieties attached directly to diphenylethyne linkers has been prepared by Tour. ${ }^{\mathrm{S} 3}$

The preparation of porphyrins bearing two different meso substituents can be readily achieved using rational approaches ${ }^{\mathrm{S} 4, \mathrm{~S} 5}$ or by a mixed-aldehyde condensation reaction. ${ }^{\mathrm{S} 6} \mathrm{~A}$ mixed-aldehyde condensation was employed to prepare porphyrins S-3 and S-4. Although not elegant, the statistical mixed-aldehyde condensation provides rapid entry to six porphyrin products, and in cases where chromatographic separation is facile, several if not all six porphyrins can be isolated from one reaction. The mixed-aldehyde condensation ${ }^{\mathrm{S} 6}$ of aldehyde S-1, benzaldehyde and pyrrole afforded target free base porphyrins $\mathbf{S - 3}$ and $\mathbf{S - 4}$, bearing one and three acetylthiomethyl linkers, respectively, in $12 \%$ and $9.5 \%$ yields (Scheme S-2). Chromatographic separation was facile given the polarity of the $S$-acetylthiomethyl group. Free base porphyrins S-3 and S-4 were each metalated using 5 equivalents of $\mathrm{Zn}(\mathrm{OAc})_{2} \cdot 2 \mathrm{H}_{2} \mathrm{O}$ in $\mathrm{CHCl}_{3} /$ methanol to yield the zinc chelates $\mathbf{S - 5}$ and $\mathbf{S - 6}$ in $92 \%$ and $89 \%$ yields, respectively.

The above reaction also yielded the cis-and trans- $\mathrm{A}_{2} \mathrm{~B}_{2}$-porphyrins. Because no separation of these two regioisomers was possible, the cis- $\mathrm{A}_{2} \mathrm{~B}_{2}$-porphyrin and trans $-\mathrm{A}_{2} \mathrm{~B}_{2}$ porphyrin were each prepared separately in a rational manner. Scheme S-3 outlines the synthesis of the cis- $\mathrm{A}_{2} \mathrm{~B}_{2}$-porphyrin S-12. 1-Acyldipyrromethane $\mathbf{S}-\mathbf{7}^{\mathrm{S} 7}$ was treated with EtMgBr followed by $p$-iodobenzoyl chloride to yield the crude 1,9-diacyldipyrromethane. We have recently developed an improved workup procedure ${ }^{\mathrm{S} 8}$ that employs selective complexation of the target 
1,9-diacyldipyrromethane with a dialkyltin reagent, resulting in a non-polar, easily separable, diacyldipyrromethane-tin complex. Following this approach, the crude mixture was treated with $\mathrm{SnBu}_{2} \mathrm{Cl}_{2}$ and TEA in $\mathrm{CH}_{2} \mathrm{Cl}_{2}$ followed by passage over a silica column. Attempts to crystallize the recovered fraction were unsuccessful as only an oil could be recovered. Accordingly, we decided to remove the tin complex. Treatment with TFA in $\mathrm{CH}_{2} \mathrm{Cl}_{2}$ followed by chromatographic workup afforded 1,9-diacyldipyrromethane S-8 in $62 \%$ yield. The difficulty in obtaining crystals of the dibutyltin species may be due to the asymmetry of the complex resulting in poor crystal packing. 1,9-Diacyldipyrromethane $\mathbf{S - 8}$ was reduced with $\mathrm{NaBH}_{4}$ and the resulting dicarbinol was condensed with 5-(4-iodophenyl)dipyrromethane (S-9) ${ }^{\mathrm{S} 9}$ under $\mathrm{Yb}(\mathrm{OTf})_{3}$ catalysis in $\mathrm{CH}_{2} \mathrm{Cl}_{2}{ }^{\mathrm{S}}$ at room temperature for $20 \mathrm{~min}$. Oxidation of the porphyrinogen with DDQ followed by zinc insertion afforded cis- $\mathrm{A}_{2} \mathrm{~B}_{2}$-porphyrin $\mathbf{S - 1 0}$ in $27 \%$ yield. No other porphyrin products arising from scrambling could be detected by laser desorption mass

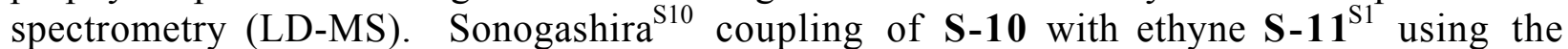
$\mathrm{Pd}_{2}(\mathrm{dba})_{3} / \mathrm{P}(o \text {-tol })_{3}$ catalytic system ${ }^{\mathrm{S} 11}$ afforded the target porphyrin $\mathbf{S - 1 2}$ in $48 \%$ yield after chromatographic workup.

Preparation of trans- $\mathrm{A}_{2} \mathrm{~B}_{2}$-porphyrin $\mathrm{S}-15$ is shown in Scheme $\mathrm{S}-4$. 1Acyldipyrromethane $\mathbf{S - 1 3}{ }^{\mathrm{S} 12}$ was treated with $\mathrm{NaBH}_{4}$ and the resulting monocarbinol was subjected to self-condensation under $\mathrm{InCl}_{3}$ catalysis in $\mathrm{CH}_{2} \mathrm{Cl}_{2}{ }^{\mathrm{S} 4}$ at room temperature for $30 \mathrm{~min}$. Oxidation of the porphyrinogen with DDQ followed by zinc insertion afforded trans $-\mathrm{A}_{2} \mathrm{~B}_{2}$ porphyrin S-14 in 24\% yield. As with the synthesis of porphyrin S-10, no scrambling was detected upon analysis by LD-MS. Sonogashira ${ }^{\mathrm{S} 10}$ coupling of porphyrin S-14 with ethyne S$\mathbf{1 1}^{\mathrm{S} 1}$ using the $\mathrm{Pd}_{2}(\mathrm{dba})_{3} / \mathrm{P}(o \text {-tol })_{3}$ catalytic system ${ }^{\mathrm{S} 11}$ afforded porphyrin $\mathbf{S}-\mathbf{1 5}$ in $57 \%$ yield after chromatographic workup.

\section{Experimental Section}

General. ${ }^{1} \mathrm{H}$ NMR spectra (400 MHz) were collected in $\mathrm{CDCl}_{3}$ (free base porphyrins) and in THF- $\mathrm{d}_{8}$ (zinc chelates). Absorption spectra were collected in $\mathrm{CH}_{2} \mathrm{Cl}_{2}$. Porphyrins were analyzed by laser desorption mass spectrometry (LD-MS) and fast bombardment mass spectrometry (FAB-MS). High-resolution mass spectrometry was carried out at greater than unit resolution. LD-MS analysis was done without a matrix. ${ }^{\text {S13 }}$ All operations involving organometallic compounds and Pd-catalyzed couplings were carried out under argon using standard Schlenk techniques. Adsorption column chromatography was performed using flash silica (60-200 mesh).

Noncommercial Samples. Compounds S-1, ${ }^{\mathrm{S} 1} \mathrm{~S}-\mathbf{7},{ }^{\mathrm{S} 7}$ S-9, ${ }^{\mathrm{S} 9} \mathbf{S - 1 1},{ }^{\mathrm{S} 1}$ and $\mathbf{S - 1 3}{ }^{\mathrm{S} 12}$ were obtained by literature procedures.

Zn(II)-meso-Tetrakis[4-[2-[4-(S-acetylthiomethyl)phenyl]ethynyl]phenyl]porphyrin (S-2). Following a general procedure, ${ }^{\mathrm{S} 2}$ a solution of $\mathrm{BF}_{3} \cdot \mathrm{O}(\mathrm{Et})_{2}(21 \square \mathrm{L}, 160 \square \mathrm{mol})$ was added to a mixture of $\mathrm{NaCl}(2.38 \mathrm{~g}, 40.7 \mathrm{mmol})$ in $\mathrm{CH}_{2} \mathrm{Cl}_{2}(136 \mathrm{~mL})$. After $5 \mathrm{~min}$, a solution of $\mathbf{S - 1}(480$ $\mathrm{mg}, 1.63 \mathrm{mmol})$ and pyrrole $(113 \square \mathrm{L}, 1.61 \mathrm{mmol})$ in $\mathrm{CH}_{2} \mathrm{Cl}_{2}(28 \mathrm{~mL})$ was added and the mixture was stirred at room temperature for $1 \mathrm{~h}$. DDQ $(278 \mathrm{mg}, 1.22 \mathrm{mmol})$ was added. Filtration over a silica column $\left(\mathrm{CH}_{2} \mathrm{Cl}_{2} / \mathrm{MeOH}, 98: 2\right)$ afforded the nearly pure free base porphyrin. A solution of the free base porphyrin in $\mathrm{CHCl}_{3}(150 \mathrm{~mL})$ was treated with a solution of $\mathrm{Zn}(\mathrm{OAc})_{2} \cdot 2 \mathrm{H}_{2} \mathrm{O}(1.00$ $\mathrm{g}, 4.56 \mathrm{mmol})$ in $\mathrm{MeOH}(15 \mathrm{~mL})$ at room temperature for $18 \mathrm{~h}$. Standard workup and column chromatography (silica, $\mathrm{CHCl}_{3}$ ) afforded a purple solid (408 mg, 70\%): ${ }^{1} \mathrm{H} \mathrm{NMR} \square 2.35(\mathrm{~s}, 12 \mathrm{H})$, $4.18(\mathrm{~s}, 8 \mathrm{H}), 7.38(\mathrm{~d}, J=8.4 \mathrm{~Hz}, 8 \mathrm{H}), 7.58(\mathrm{~d}, J=7.6 \mathrm{~Hz}, 8 \mathrm{H}), 7.92(\mathrm{~d}, J=8.4 \mathrm{~Hz}, 8 \mathrm{H}), 8.22(\mathrm{~d}$, $J=8.0 \mathrm{~Hz}, 8 \mathrm{H}), 8.90(\mathrm{~s}, 8 \mathrm{H})$; LD-MS obsd $1427.3\left[\mathrm{M}^{+}\right], 1353.2\left[(\mathrm{M}-\mathrm{SAc})^{+}\right], 1279.9[(\mathrm{M}-2 \mathrm{x}$ $\left.\mathrm{SAc})^{+}\right], 1204.1\left[(\mathrm{M}-3 \times \mathrm{SAc})^{+}\right], 1128.2\left[(\mathrm{M}-4 \mathrm{x} \mathrm{SAc})^{+}\right]$; FAB-MS no signal observed, calcd

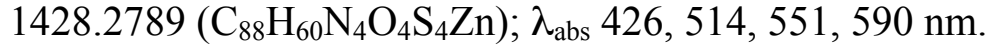


5-[4-[2-[4-(S-Acetylthiomethyl)phenyl]ethynyl]phenyl]-10,15,20-triphenylporphyrin ( S - 3) and 5, 1 0, 15 - T r is [ 4 - [ 2 - [ 4 - ( $S$ - acetylthiomethyl)phenyl]ethynyl]phenyl]-20phenylporphyrin (S-4). Following a general procedure for mixed-aldehyde condensation ${ }^{\mathrm{S} 6}$ with $\mathrm{BF}_{3} \cdot \mathrm{O}(\mathrm{Et})_{2}$ cocatalysis, ${ }^{\mathrm{S} 14}$ a solution of $\mathbf{S}-1$ ( $\left.250 \mathrm{mg}, 0.85 \mathrm{mmol}\right)$, benzaldehyde $(90.2 \mathrm{~g}, 0.85$ $\mathrm{mmol})$, pyrrole $(120 \square \mathrm{L}, 1.70 \mathrm{mmol})$ and $\mathrm{BF}_{3} \cdot \mathrm{O}(\mathrm{Et})_{2}\left(204 \square \mathrm{L}, 2.5 \mathrm{M}\right.$ in $\left.\mathrm{CHCl}_{3}\right)$ in $\mathrm{CHCl}_{3}(170$ $\mathrm{mL}$ ) was stirred at room temperature for $1.5 \mathrm{~h}$. DDQ (289 $\mathrm{mg}, 1.27 \mathrm{mmol}$ ) was added. The mixture was filtered through a pad of silica $\left(\mathrm{CH}_{2} \mathrm{Cl}_{2}\right)$ followed by column chromatography [silica, $\mathrm{CH}_{2} \mathrm{Cl}_{2} /$ hexanes (4:1)] to give clean separation of four porphyrins and a mixture of cis$\mathrm{A}_{2} \mathrm{~B}_{2}$ - and trans- $\mathrm{A}_{2} \mathrm{~B}_{2}$-porphyrins. Porphyrin $\mathbf{S - 3}$ eluted as the second purple band (41.0 mg, 12\%): ${ }^{1} \mathrm{H}$ NMR $\square-2.77(\mathrm{~s}, 2 \mathrm{H}), 2.40(\mathrm{~s}, 3 \mathrm{H}), 4.18(\mathrm{~s}, 2 \mathrm{H}), 7.36(\mathrm{~d}, J=8.0 \mathrm{~Hz}, 2 \mathrm{H}), 7.61(\mathrm{~d}, J=$ $8.4 \mathrm{~Hz}, 2 \mathrm{H}), 7.72-7.81(\mathrm{~m}, 9 \mathrm{H}), 7.92(\mathrm{~d}, J=8.0 \mathrm{~Hz}, 2 \mathrm{H}), 8.21(\mathrm{~d}, J=8.0 \mathrm{~Hz}, 8 \mathrm{H}), 8.85(\mathrm{~s}, 4 \mathrm{H})$, $8.86(\mathrm{~s}, 4 \mathrm{H})$; LD-MS obsd $803.3\left[\mathrm{M}^{+}\right], 727.3\left[(\mathrm{M}-\mathrm{SAc})^{+}\right]$; FAB-MS obsd 802.2781, calcd 802.2766 $\left(\mathrm{C}_{55} \mathrm{H}_{38} \mathrm{~N}_{4} \mathrm{OS}\right)$; - $\mathrm{abs}_{\mathrm{bs}} 422,515,550,592,648 \mathrm{~nm}$. Porphyrin S-4 eluted as the fourth purple band (47.4 mg, 9.5\%): ${ }^{1} \mathrm{H}$ NMR $\square-2.78(\mathrm{~s}, 2 \mathrm{H}), 2.40(\mathrm{~s}, 9 \mathrm{H}), 4.18(\mathrm{~s}, 6 \mathrm{H}), 7.36(\mathrm{~d}, J=$ $8.4 \mathrm{~Hz}, 6 \mathrm{H}), 7.61(\mathrm{~d}, J=8.0 \mathrm{~Hz}, 6 \mathrm{H}), 7.74-7.80(\mathrm{~m}, 3 \mathrm{H}), 7.93(\mathrm{~d}, J=8.0 \mathrm{~Hz}, 6 \mathrm{H}), 8.21(\mathrm{~d}, J=$ $8.0 \mathrm{~Hz}, 8 \mathrm{H}), 8.87(\mathrm{~s}, 4 \mathrm{H}), 8.88(\mathrm{~s}, 4 \mathrm{H})$; LD-MS obsd $1180.2\left[\mathrm{M}^{+}\right], 1105.2\left[(\mathrm{M}-\mathrm{SAc})^{+}\right], 1030.9$ $\left[\left(\mathrm{M}-2 \times \mathrm{SAc}^{+}\right], 955.6\left[(\mathrm{M}-3 \mathrm{x} \mathrm{SAc})^{+}\right]\right.$; FAB-MS obsd 1178.333, calcd 1178.336 $\left(\mathrm{C}_{77} \mathrm{H}_{54} \mathrm{~N}_{4} \mathrm{O}_{3} \mathrm{~S}_{3}\right)$; - abs $424,518,553,594,650 \mathrm{~nm}$.

\section{Zn(II)-5-[4-[2-[4-(S-Acetylthiomethyl)phenyl]ethynyl]phenyl]-10,15,20-}

triphenylporphyrin (S-5). A solution of $\mathbf{S - 3}(25.0 \mathrm{mg}, 31.1 \square \mathrm{mol})$ in $\mathrm{CHCl}_{3} / \mathrm{MeOH}(6 \mathrm{~mL}, 2: 1)$ was treated with a solution of $\mathrm{Zn}(\mathrm{OAc})_{2} \cdot 2 \mathrm{H}_{2} \mathrm{O}(34.1 \mathrm{mg}, 156 \square \mathrm{mol})$ in $\mathrm{MeOH}(1 \mathrm{~mL})$. Column chromatography (silica, $\mathrm{CH}_{2} \mathrm{Cl}_{2}$ ) and crystallization afforded pink crystals $(24.8 \mathrm{mg}, 92 \%):{ }^{1} \mathrm{H}$ NMR $\square 2.35(\mathrm{~s}, 3 \mathrm{H}), 4.18(\mathrm{~s}, 2 \mathrm{H}), 7.39(\mathrm{~d}, J=8.0 \mathrm{~Hz}, 2 \mathrm{H}), 7.58(\mathrm{~d}, J=8.0 \mathrm{~Hz}, 2 \mathrm{H}), 7.72-7.78$ $(\mathrm{m}, 9 \mathrm{H}), 7.89-7.92(\mathrm{~m}, 2 \mathrm{H}), 8.18-8.23(\mathrm{~m}, 8 \mathrm{H}), 8.82-8.88(\mathrm{~m}, 8 \mathrm{H})$; LD-MS obsd $866.6\left[\mathrm{M}^{+}\right]$, $792.2\left[(\mathrm{M}-\mathrm{SAc})^{+}\right]$; FAB-MS obsd 864.1890, calcd 864.1901 $\left(\mathrm{C}_{55} \mathrm{H}_{36} \mathrm{~N}_{4} \mathrm{OSZn}\right)$; $\square_{a b s} 421,512$, $548,587 \mathrm{~nm}$.

Zn(II)-5,10,15-Tris[4-[2-[4-(S-acetylthiomethyl)phenyl]ethynyl]phenyl]-20-

phenylporphyrin (S-6). A solution of S-4 (25.0 mg, $21.2 \square \mathrm{mol})$ in $\mathrm{CHCl}_{3} / \mathrm{MeOH}(6 \mathrm{~mL}, 2: 1)$ was treated with a solution of $\mathrm{Zn}(\mathrm{OAc})_{2} \cdot 2 \mathrm{H}_{2} \mathrm{O}(23.3 \mathrm{mg}, 106 \square \mathrm{mol})$ in $\mathrm{MeOH}(1 \mathrm{~mL})$. Column chromatography (silica, $\mathrm{CH}_{2} \mathrm{Cl}_{2}$ ) and crystallization afforded pink crystals $(23.5 \mathrm{mg}, 89 \%):{ }^{1} \mathrm{H}$ NMR $\square 2.35$ (s, 9H), $4.18(\mathrm{~s}, 6 \mathrm{H}), 7.39$ (d, $J=8.4 \mathrm{~Hz}, 6 \mathrm{H}), 7.58(\mathrm{~d}, J=8.0 \mathrm{~Hz}, 6 \mathrm{H}), 7.74-7.76$ $(\mathrm{m}, 3 \mathrm{H}), 7.89-7.93(\mathrm{~m}, 6 \mathrm{H}), 8.18-8.23(\mathrm{~m}, 8 \mathrm{H}), 8.86-8.90(\mathrm{~m}, 8 \mathrm{H})$; LD-MS obsd 1241.1 [M $\left.^{+}\right]$, $1166.2[(\mathrm{M}-\mathrm{SAc})], 1091.2\left[(\mathrm{M}-2 \times \mathrm{SAc})^{+}\right], 1013.1\left[(\mathrm{M}-3 \times \mathrm{SAc})^{+}\right]$; FAB-MS obsd 1240.26, calcd $1240.2493\left(\mathrm{C}_{77} \mathrm{H}_{52} \mathrm{~N}_{4} \mathrm{O}_{3} \mathrm{~S}_{3} \mathrm{Zn}\right)$; - abs $424,513,550,590 \mathrm{~nm}$.

1-Benzoyl-9-(4-iodobenzoyl)-5-phenyldipyrromethane (S-8). Following a general procedure, ${ }^{\mathrm{S} 5, \mathrm{~S} 8}$ a solution of $\mathbf{S - 7}(3.00 \mathrm{~g}, 9.19 \mathrm{mmol})$ in toluene $(92 \mathrm{~mL})$ at room temperature under argon was treated with $\operatorname{EtMgBr}(55 \mathrm{~mL}, 55 \mathrm{mmol}, 1.0 \mathrm{M}$ solution in THF). A sample of 4-iodobenzoyl chloride $\left(7.35 \mathrm{~g}, 27.6 \mathrm{mmol}\right.$ ) was added. After $30 \mathrm{~min}$, saturated aqueous $\mathrm{NH}_{4} \mathrm{Cl}$ and ethyl acetate were added. The organic layer was collected, dried $\left(\mathrm{Na}_{2} \mathrm{SO}_{4}\right)$, and filtered. The filtrate was concentrated. The crude oil was dissolved in $\mathrm{CH}_{2} \mathrm{Cl}_{2}(50 \mathrm{~mL})$ and samples of TEA $(3.76 \mathrm{~mL}, 27.6 \mathrm{mmol})$ and $\mathrm{Bu}_{2} \mathrm{SnCl}_{2}(2.79 \mathrm{~g}, 9.19 \mathrm{mmol})$ were added. After $20 \mathrm{~min}$, the mixture was passed over a silica column $\left(\mathrm{CH}_{2} \mathrm{Cl}_{2}\right)$. The fractions containing the tin complex were collected and concentrated. Attempted crystallization from $\mathrm{Et}_{2} \mathrm{O} / \mathrm{MeOH}$ was unsuccessful. The tin complex was then dissolved in $\mathrm{CH}_{2} \mathrm{Cl}_{2}(50 \mathrm{~mL})$ and a sample of TFA $(2.2 \mathrm{~mL}, 28 \mathrm{mmol})$ was added. After $30 \mathrm{~min}$, the reaction mixture was concentrated. Column chromatography [silica, $\mathrm{CH}_{2} \mathrm{Cl}_{2} /$ EtOAc (95:5)] afforded a pink solid (3.17 g, 62\%): $\mathrm{mp} 66-70{ }^{\circ} \mathrm{C} ;{ }^{1} \mathrm{H} \mathrm{NMR} \square 5.66$ (s, 1H), 6.00-6.04 (m, 2H), 6.59-6.65 (m, 2H), 7.34-7.53 (m, 10H), 7.53-7.79 (m, 4H), 11.04 (br s, 
$2 \mathrm{H}) ;{ }^{13} \mathrm{C}$ NMR $\square 44.9,99.3,111.5,111.7,121.5,121.7,127.5,128.0,128.7,128.8,129.4,129.6$, $130.5,130.8,131.1,131.9,137.2,137.3,137.77,137.82,140.0,141.5,142.0,162.9,183.7$, 184.9; FAB-MS obsd 557.0751, calcd 557.0646 [(M + H $\left.)^{+}\right]\left(\mathrm{M}=\mathrm{C}_{29} \mathrm{H}_{21} \mathrm{IN}_{2} \mathrm{O}_{2}\right)$.

Zn(II)-5,10-(4-Iodophenyl)-15,20-diphenylporphyrin (S-10). Following a general procedure, ${ }^{\mathrm{S} 5}$ a solution of $\mathbf{S - 8}(1.50 \mathrm{~g}, 2.70 \mathrm{mmol})$ in THF/MeOH $[50 \mathrm{~mL},(10: 1)]$ was reduced with $\mathrm{NaBH}_{4}(2.04 \mathrm{~g}, 53.9 \mathrm{mmol})$. The resulting dicarbinol was condensed with S-9 $(940 \mathrm{mg}$, $2.70 \mathrm{mmol})$ in $\mathrm{CH}_{2} \mathrm{Cl}_{2}(540 \mathrm{~mL})$ containing $\mathrm{Yb}(\mathrm{OTf})_{3}(1.07 \mathrm{~g}, 1.73 \mathrm{mmol})$ as acid catalyst ${ }^{\mathrm{S}}$ at room temperature for $20 \mathrm{~min}$. DDQ $(1.84 \mathrm{~g}, 8.10 \mathrm{mmol})$ was added. The mixture was passed over a silica column $\left(\mathrm{CH}_{2} \mathrm{Cl}_{2}\right)$ and the porphyrin-containing fraction was collected and concentrated. The free base porphyrin was dissolved in $\mathrm{CHCl}_{3}(200 \mathrm{~mL})$ and treated with a solution of $\mathrm{Zn}(\mathrm{OAc})_{2} \cdot 2 \mathrm{H}_{2} \mathrm{O}(592 \mathrm{mg}, 2.70 \mathrm{mmol})$ in $\mathrm{MeOH}(15 \mathrm{~mL})$ at room temperature for 19 h. Standard workup including chromatography (silica, $\left.\mathrm{CHCl}_{3}\right)$ afforded a purple solid $(681 \mathrm{mg}$, 27\%): ${ }^{1} \mathrm{H}$ NMR $\square 7.73-7.81(\mathrm{~m}, 6 \mathrm{H}), 7.95(\mathrm{~d}, J=8.0 \mathrm{~Hz}, 4 \mathrm{H}), 8.08(\mathrm{~d}, J=8.0 \mathrm{~Hz}, 4 \mathrm{H}), 8.21(\mathrm{dd}$, $8.0 \mathrm{~Hz}, 1.5 \mathrm{~Hz}, 4 \mathrm{H}), 8.93-8.98$ (m, 8H); LD-MS obsd $928.2\left[\mathrm{M}^{+}\right]$; 813.3 [(M - I $\left.)^{+}\right]$; FAB-MS

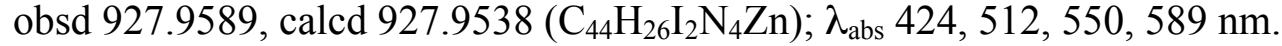

Zn(II)-5,10-Bis[4-[2-[4-(S-acetylthiomethyl)phenyl]ethynyl]phenyl]-15,20-

diphenylporphyrin (S-12). Following a general procedure with slight modification, ${ }^{\mathrm{S} 11}$ samples of S-10 (150 mg, $161 \square \mathrm{mol}), \mathrm{Pd}_{2}(\mathrm{dba})_{3}$ (44 mg, $\left.48 \square \mathrm{mol}\right), \mathrm{P}(o \text {-tol) })_{3}(59 \mathrm{mg}, 190 \square \mathrm{mol})$, and S-11 $(153 \mathrm{mg}, 0.804 \mathrm{mmol})$ in THF/TEA [60 $\mathrm{mL}(5: 1)]$ were heated to $40{ }^{\circ} \mathrm{C}$ for $40 \mathrm{~h}$. The mixture was concentrated. Column chromatography [silica, $\mathrm{CHCl}_{3}$ /hexanes $(3: 1) \square \mathrm{CHCl}_{3} \quad$ $\mathrm{CHCl}_{3} /$ EtOAc (19:1)] followed by washing the recovered solid with $\mathrm{MeOH}$ afforded a purple solid (82 mg, 48\%): ${ }^{1} \mathrm{H}$ NMR $\mathrm{T} 2.49(\mathrm{~s}, 6 \mathrm{H}), 4.18(\mathrm{~s}, 4 \mathrm{H}), 7.38-7.40(\mathrm{~m}, 4 \mathrm{H}), 7.58(\mathrm{~d}, J=8.0$ $\mathrm{Hz}, 4 \mathrm{H}), 7.74-7.75(\mathrm{~m}, 6 \mathrm{H}), 7.91(\mathrm{~d}, J=8.0 \mathrm{~Hz}, 4 \mathrm{H}), 8.19-8.24(\mathrm{~m}, 8 \mathrm{H}), 8.85-8.90(\mathrm{~m}, 8 \mathrm{H})$; LD-MS obsd 1052.5; FAB-MS obsd 1052.2218, calcd $1052.2197\left(\mathrm{C}_{66} \mathrm{H}_{44} \mathrm{~N}_{4} \mathrm{O}_{2} \mathrm{~S}_{2} \mathrm{Zn}\right)$; $\square_{\text {abs }} 426$, $551,591 \mathrm{~nm}$.

Zn(II)-5,15-(4-Iodophenyl)-10,20-diphenylporphyrin (S-14). Following a general procedure, ${ }^{\mathrm{S} 5}$ a solution of $\mathbf{S - 1 3}(1.40 \mathrm{~g}, 3.10 \mathrm{mmol})$ in THF/MeOH [60 $\left.\mathrm{mL},(10: 1)\right]$ was reduced with $\mathrm{NaBH}_{4}(2.93 \mathrm{~g}, 77.4 \mathrm{mmol})$. The resulting monocarbinol was subjected to selfcondensation in $\mathrm{CH}_{2} \mathrm{Cl}_{2}(620 \mathrm{~mL})$ containing $\mathrm{InCl}_{3}(44 \mathrm{mg}, 198 \square \mathrm{mol})$ as catalyst ${ }^{\mathrm{S} 5}$ at room temperature for $30 \mathrm{~min}$. DDQ $(1.41 \mathrm{~g}, 6.20 \mathrm{mmol})$ was added. The mixture was passed over a silica column $\left(\mathrm{CH}_{2} \mathrm{Cl}_{2}\right)$ and the porphyrin-containing fraction was collected and concentrated. The free base porphyrin was dissolved in $\mathrm{CHCl}_{3}(150 \mathrm{~mL})$ and treated with a solution of $\mathrm{Zn}(\mathrm{OAc})_{2} \cdot 2 \mathrm{H}_{2} \mathrm{O}(680 \mathrm{mg}, 3.10 \mathrm{mmol})$ in $\mathrm{MeOH}(15 \mathrm{~mL})$ at room temperature for $2 \mathrm{~h}$. Standard workup including chromatography (silica, $\mathrm{CHCl}_{3}$ ) afforded a purple solid $(347 \mathrm{mg}, 24 \%):{ }^{1} \mathrm{H}$ NMR $\square 7.74-7.80(\mathrm{~m}, 6 \mathrm{H}), 7.95(\mathrm{~d}, J=8.0 \mathrm{~Hz}, 4 \mathrm{H}), 8.09(\mathrm{~d}, J=8.0 \mathrm{~Hz}, 4 \mathrm{H}), 8.21(\mathrm{~d}, 8.0 \mathrm{~Hz}$, 4H), 8.93-8.97 (m, 8H); LD-MS obsd 928.1; FAB-MS obsd 927.9534, calcd 927.9538 $\left(\mathrm{C}_{44} \mathrm{H}_{26} \mathrm{I}_{2} \mathrm{~N}_{4} \mathrm{Zn}\right)$; $\square_{\text {abs }} 424,512,550,590 \mathrm{~nm}$.

\section{Zn(II)-5,15-Bis[4-[2-[4-(S-acetylthiomethyl)phenyl]ethynyl]phenyl]-10,20-}

diphenylporphyrin (S-15). Following a general procedure with slight modification, ${ }^{\mathrm{S} 11}$ samples of S-14 (150 mg, $161 \square \mathrm{mol}), \mathrm{Pd}_{2}(\mathrm{dba})_{3}(44 \mathrm{mg}, 48 \square \mathrm{mol}), \mathrm{P}(\mathrm{o} \text {-tol) })_{3}(59 \mathrm{mg}, 190 \square \mathrm{mol})$, and S-11 (153 mg, $0.804 \mathrm{mmol}$ ) in THF/TEA [60 mL, (5:1)] were heated to $40{ }^{\circ} \mathrm{C}$ for $40 \mathrm{~h}$. The mixture was concentrated. Column chromatography [silica, $\mathrm{CHCl}_{3} /$ hexanes $(3: 1) \square \mathrm{CHCl}_{3} \quad \square$ $\mathrm{CHCl}_{3} /$ EtOAc (19:1)] followed by washing the recovered solid with $\mathrm{MeOH}$ afforded a purple solid (96 mg, 57\%): ${ }^{1} \mathrm{H}$ NMR $\square 2.49(\mathrm{~s}, 6 \mathrm{H}), 4.19(\mathrm{~s}, 4 \mathrm{H}), 7.38-7.40(\mathrm{~m}, 4 \mathrm{H}), 7.58(\mathrm{~d}, J=8.0$ $\mathrm{Hz}, 4 \mathrm{H}), 7.74-7.75$ (m, 6H), $7.91(\mathrm{~d}, J=8.0 \mathrm{~Hz}, 4 \mathrm{H}), 8.20-8.23(\mathrm{~m}, 8 \mathrm{H}), 8.85-8.90(\mathrm{~m}, 8 \mathrm{H})$; LD-MS obsd 1052.4; FAB-MS obsd 1052.2260, calcd $1052.2197\left(\mathrm{C}_{66} \mathrm{H}_{44} \mathrm{~N}_{4} \mathrm{O}_{2} \mathrm{~S}_{2} \mathrm{Zn}\right)$; $\square_{\text {abs }} 427$, $551,591 \mathrm{~nm}$. 


\section{References}

(S1) Gryko, D. T.; Clausen, C.; Roth, K. M.; Dontha, N.; Bocian, D. F.; Kuhr, W. G.; Lindsey, J. S. J. Org. Chem. 2000, 65, 7345-7355.

(S2) Geier, G. R., III.; Riggs, J. A.; Lindsey, J. S. J. Porphyrins Phthalocyanines 2001, 5, 681-690.

(S3) Jagessar, R. C.; Tour, J. M. Org. Lett. 2000, 2, 111-113.

(S4) Geier, G. R., III.; Callinan, J. B.; Rao, P. D.; Lindsey, J. S. J. Porphyrins Phthalocyanines 2001, 5, 810-823.

(S5) Rao, P. D.; Dhanalekshmi, S.; Littler, B. J.; Lindsey, J. S. J. Org. Chem. 2000, 65, 7323-7344.

(S6) Lindsey, J. S.; Prathapan, S.; Johnson, T. E.; Wagner, R. W. Tetrahedron 1994, 50, 8941-8968.

(S7) Geier, G. R., III.; Lindsey, J. S. J. Chem. Soc., Perkin Trans. 2 2001, 5, 687-700.

(S8) Tamaru, S.-i.; Yu, L.; Youngblood, W. J.; Muthukumaran, K.; Taniguchi, M.; Lindsey, J. S. J. Org. Chem. 2004, 69, 765-777.

(S9) Lee, C.-H.; Lindsey, J. S. Tetrahedron 1994, 50, 11427-11440.

(S10) Sonogashira, K.; Tohda, Y.; Hagihara, N. Tetrahedron Lett. 1975, 4467-4470.

(S11) (a) Wagner, R. W.; Johnson, T. E.; Li, F.; Lindsey, J. S. J. Org. Chem. 1995, 60, 5266-5273. (b) Wagner, R. W.; Ciringh, Y.; Clausen, C.; Lindsey, J. S. Chem. Mater. 1999, 11, 2974-2983.

(S12) Rao, P. D.; Littler, B. J.; Geier, G. R., III.; Lindsey, J. S. J. Org. Chem. 2000, 65, 1084-1092.

(S13) (a) Fenyo, D.; Chait, B. T.; Johnson, T. E.; Lindsey, J. S. J. Porphyrins Phthalocyanines 1997, 1, 93-99. (b) Srinivasan, N.; Haney, C. A.; Lindsey, J. S.; Zhang, W.; Chait, B. T. J. Porphyrins Phthalocyanines 1999, 3, 283-291.

(S14) Lindsey, J. S.; Wagner, R. W. J. Org. Chem. 1989, 54, 828-836. 
Scheme S1

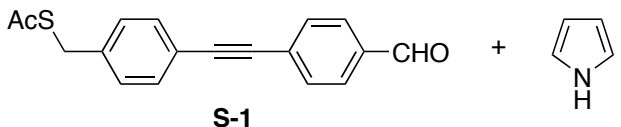

S-1

$$
70 \% \mid \begin{aligned}
& \text { (1) } \mathrm{BF}_{3} \cdot \mathrm{O}(\mathrm{Et})_{2}, \mathrm{NaCl}, \mathrm{CH}_{2} \mathrm{Cl}_{2} \\
& \text { (2) } \mathrm{DDQ} \\
& \text { (3) } \mathrm{Zn}(\mathrm{OAc})_{2} \cdot 2 \mathrm{H}_{2} \mathrm{O}
\end{aligned}
$$

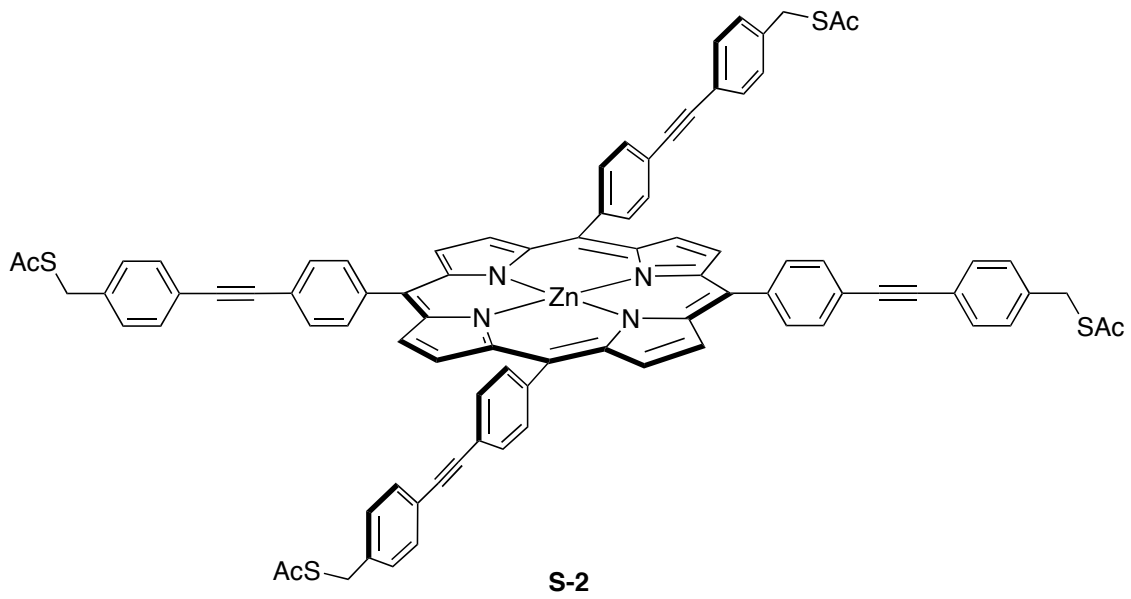




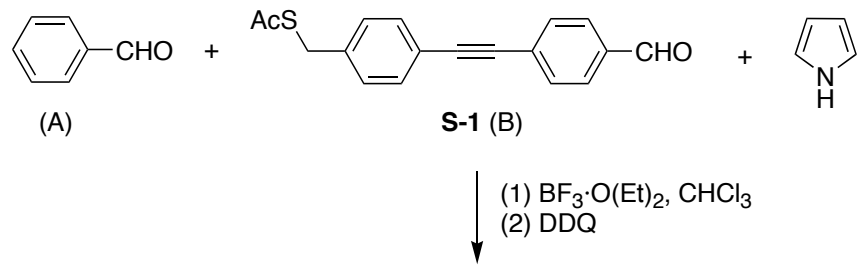

mixture of porphyrins

meso-tetraphenylporphyrin $\left(\mathrm{A}_{4}\right)$

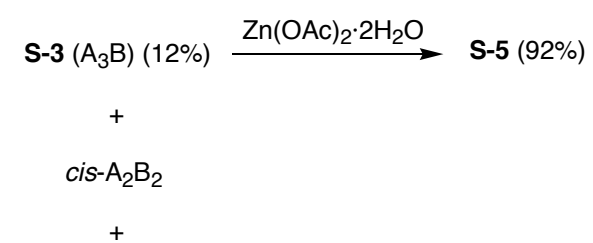

trans $-\mathrm{A}_{2} \mathrm{~B}_{2}$

$+$

$\mathrm{S}-4\left(\mathrm{AB}_{3}\right)(9.5 \%) \stackrel{\mathrm{Zn}(\mathrm{OAc})_{2} \cdot 2 \mathrm{H}_{2} \mathrm{O}}{\longrightarrow} \mathrm{S}-6(89 \%)$

$+$

$\mathrm{B}_{4}$

(only S-3 and S-4 were isolated)

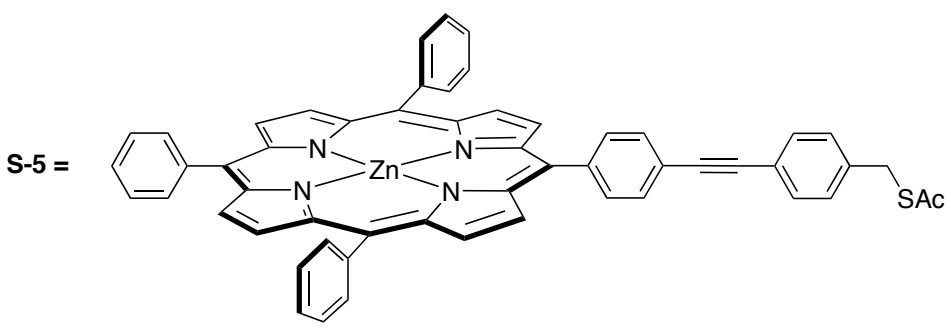

S-6 =

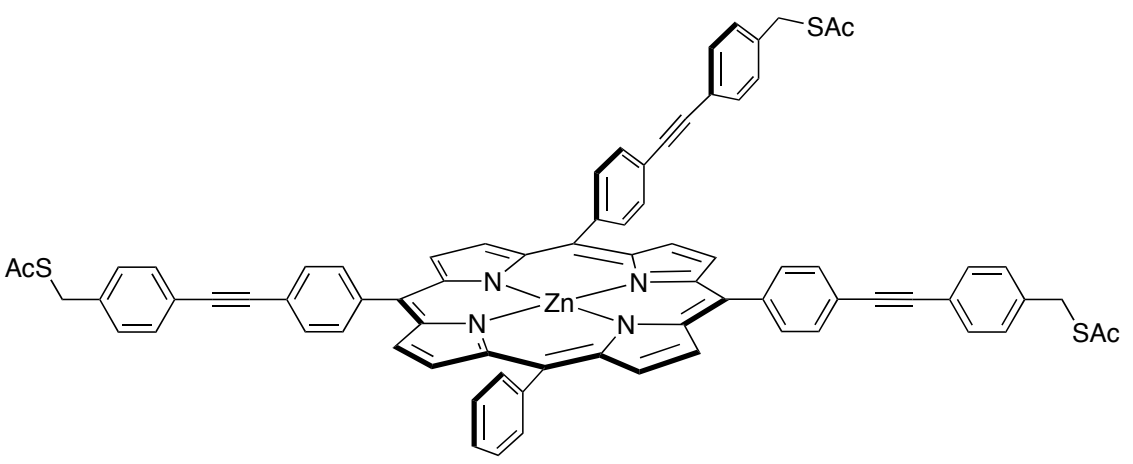




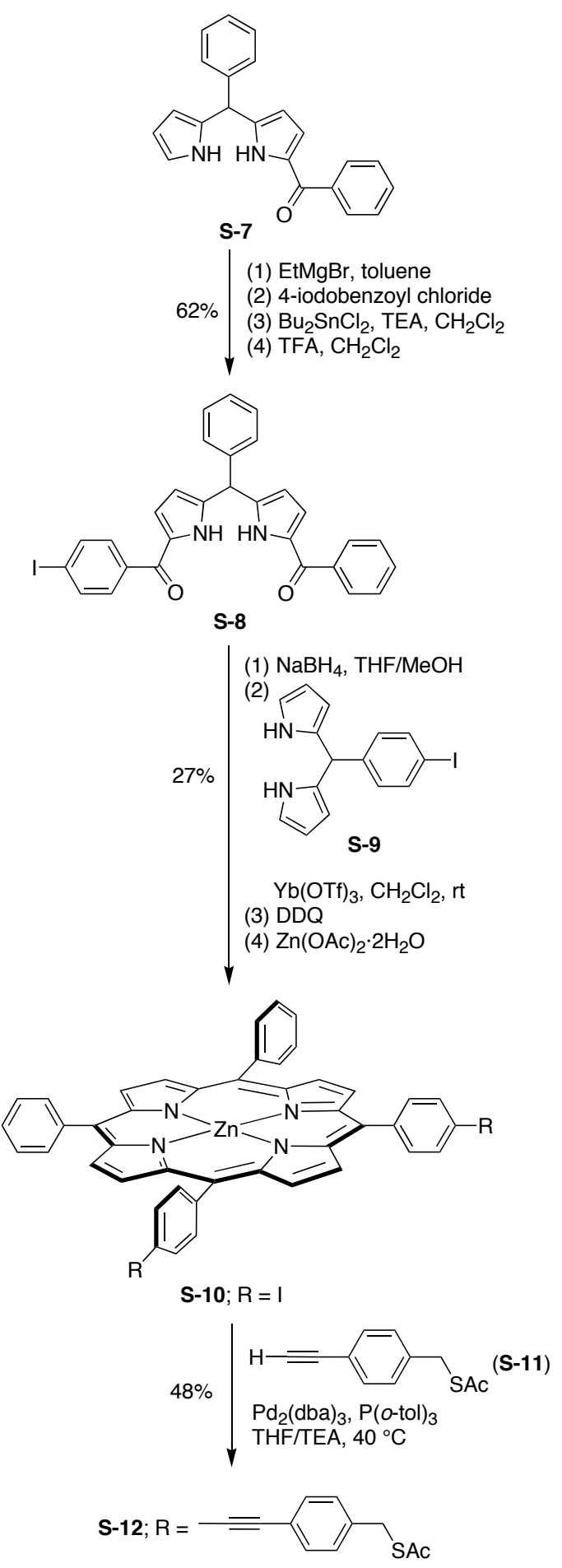


Scheme S4

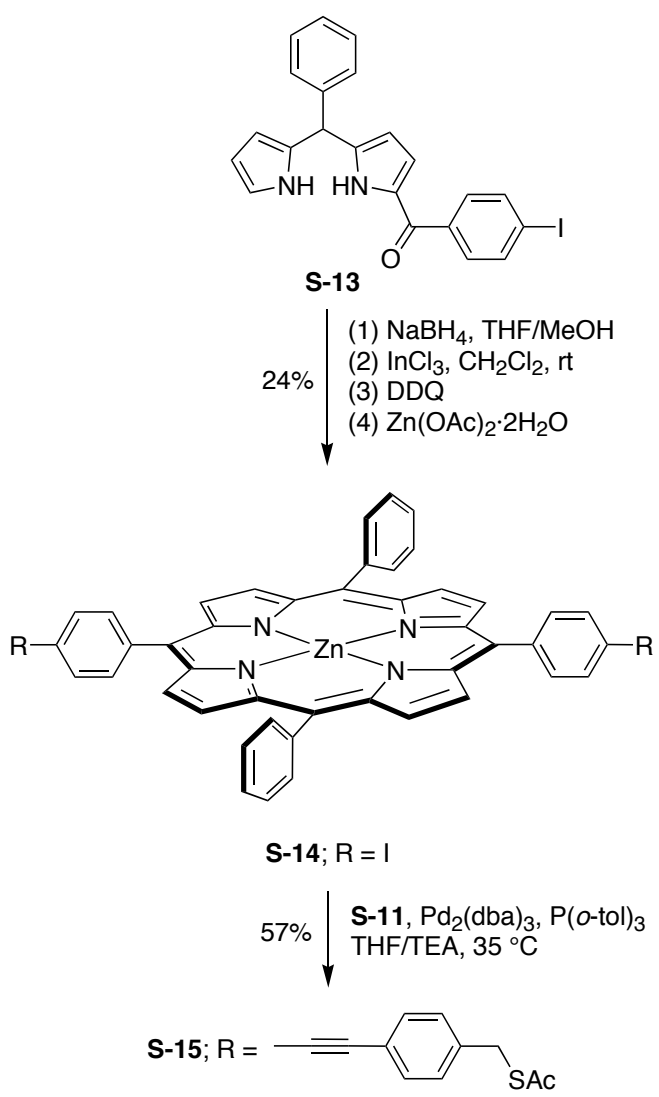




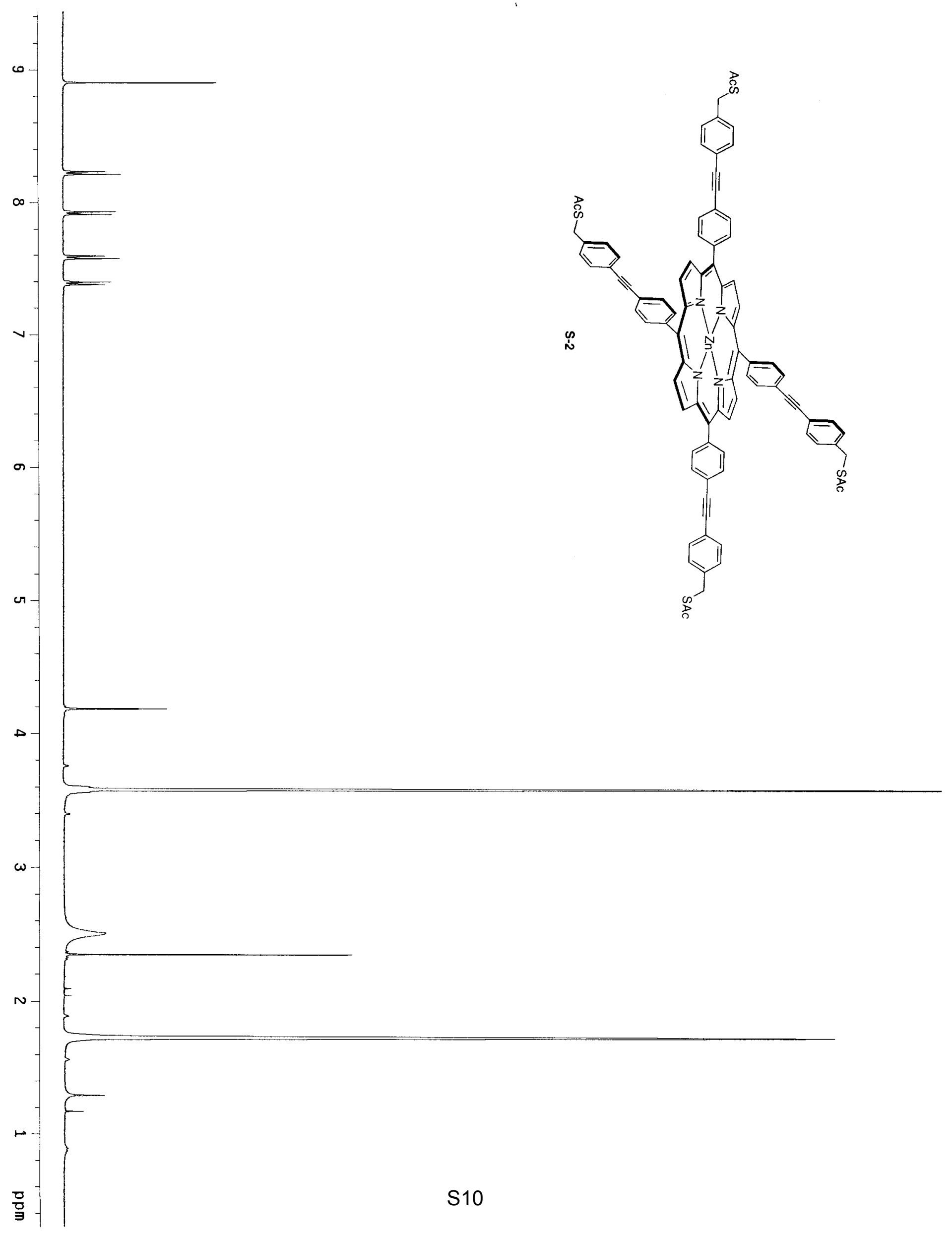




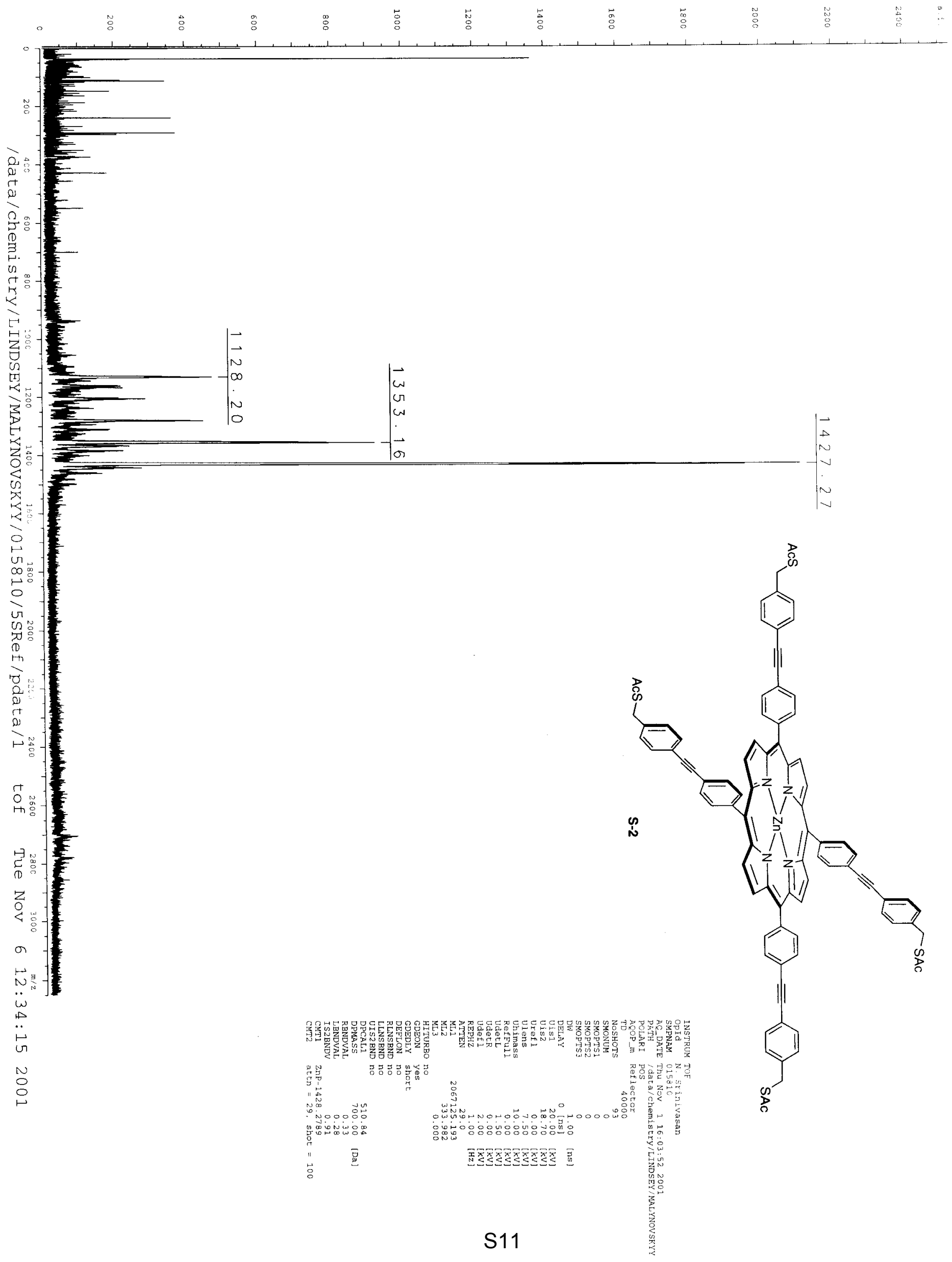




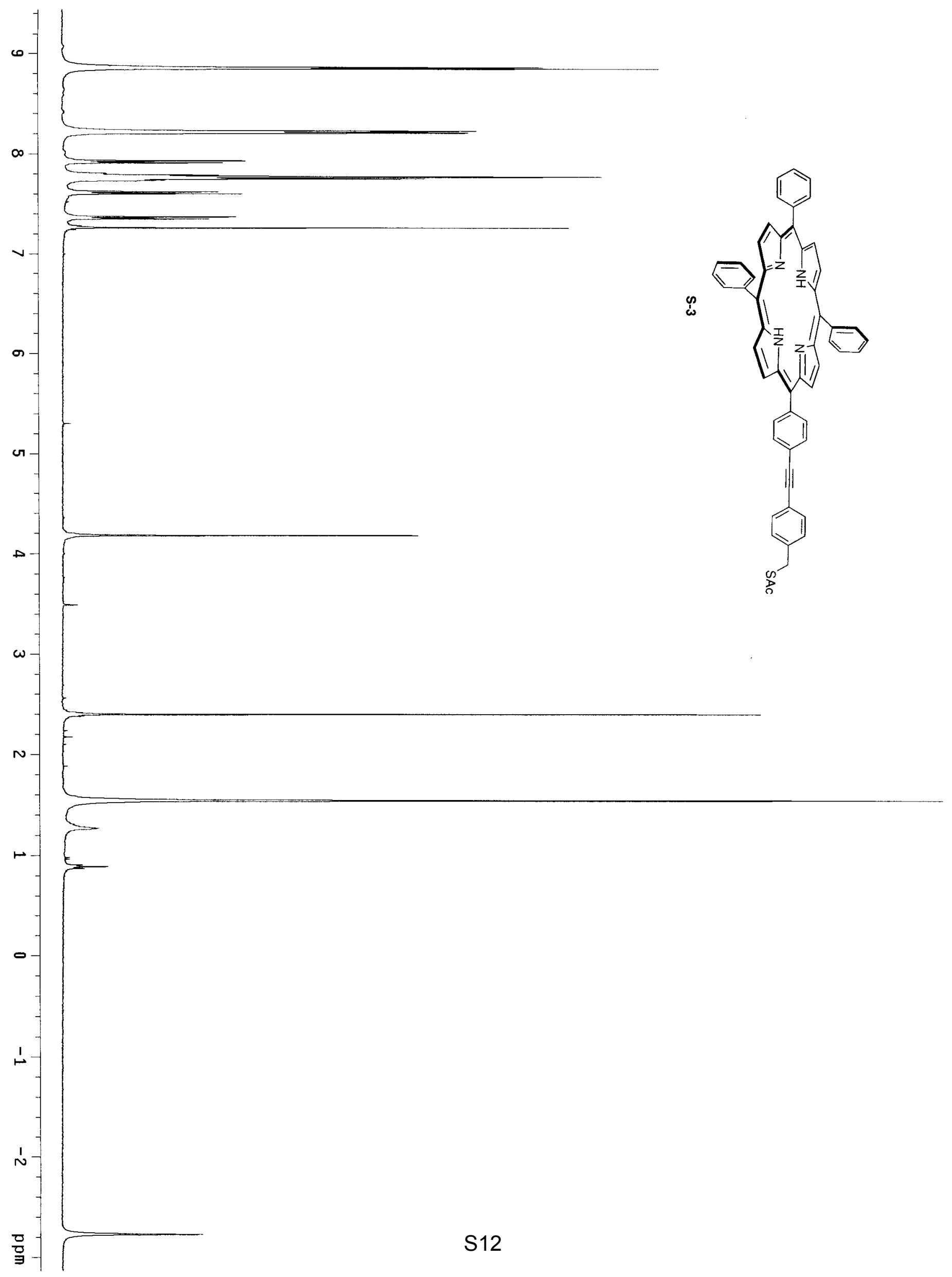




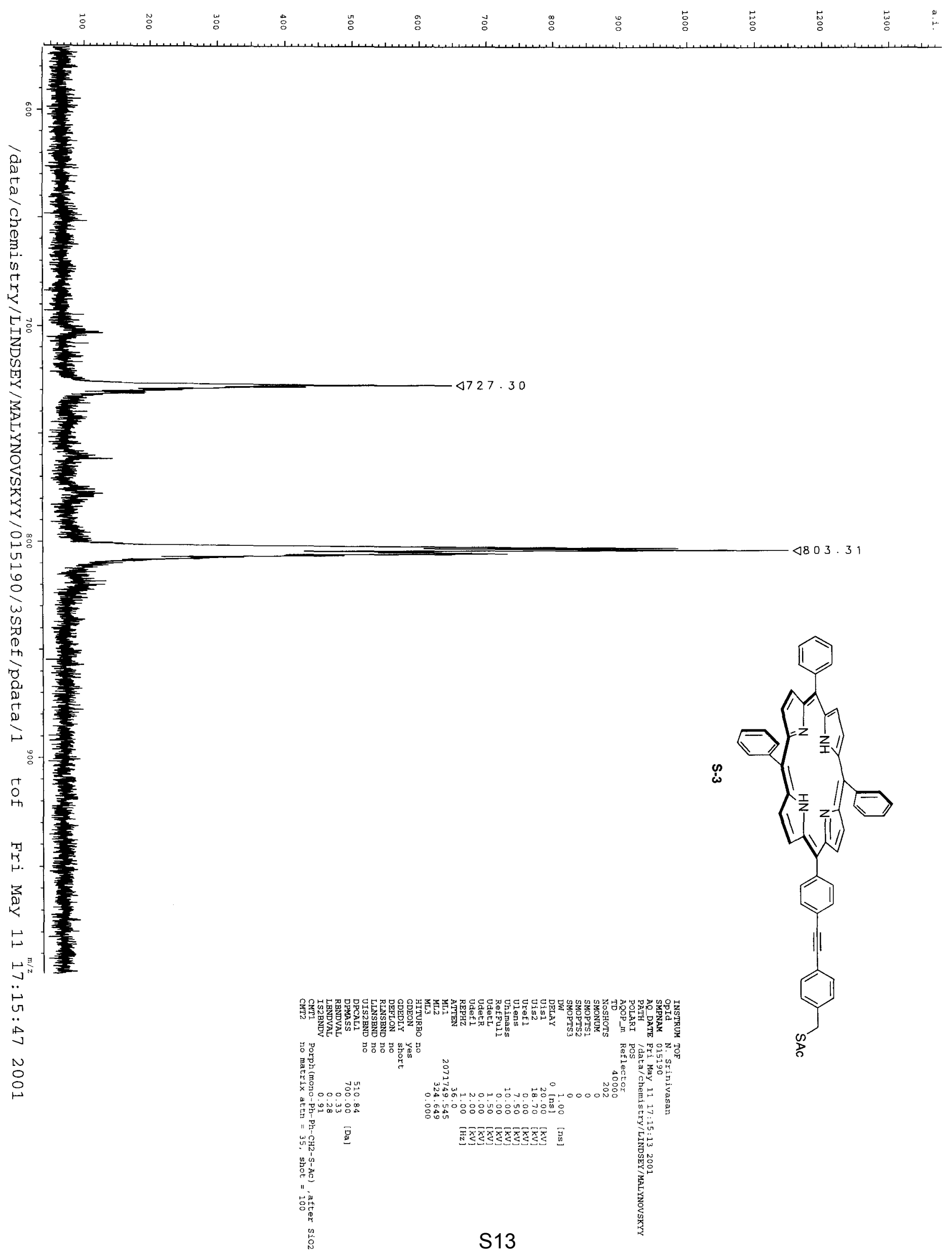




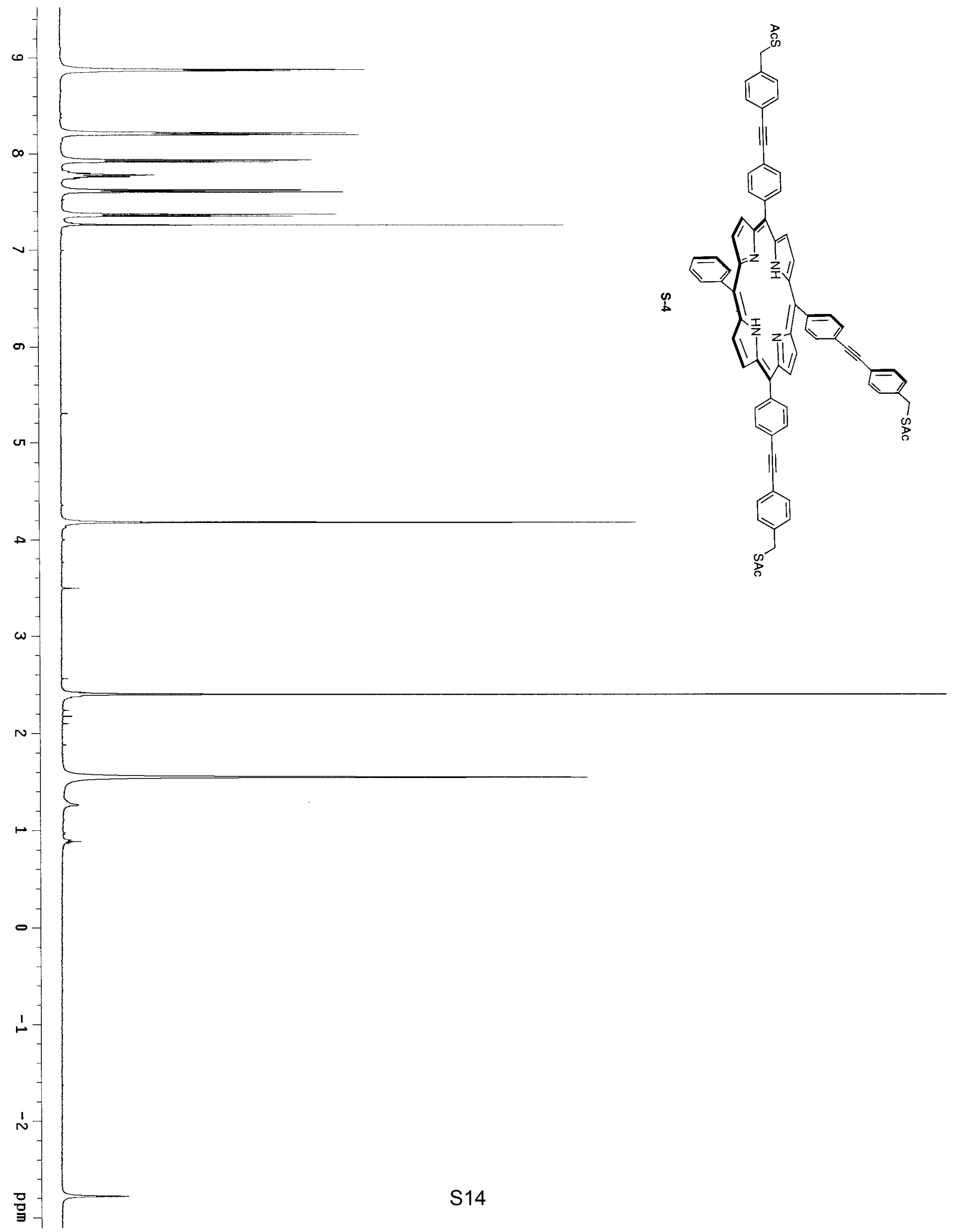




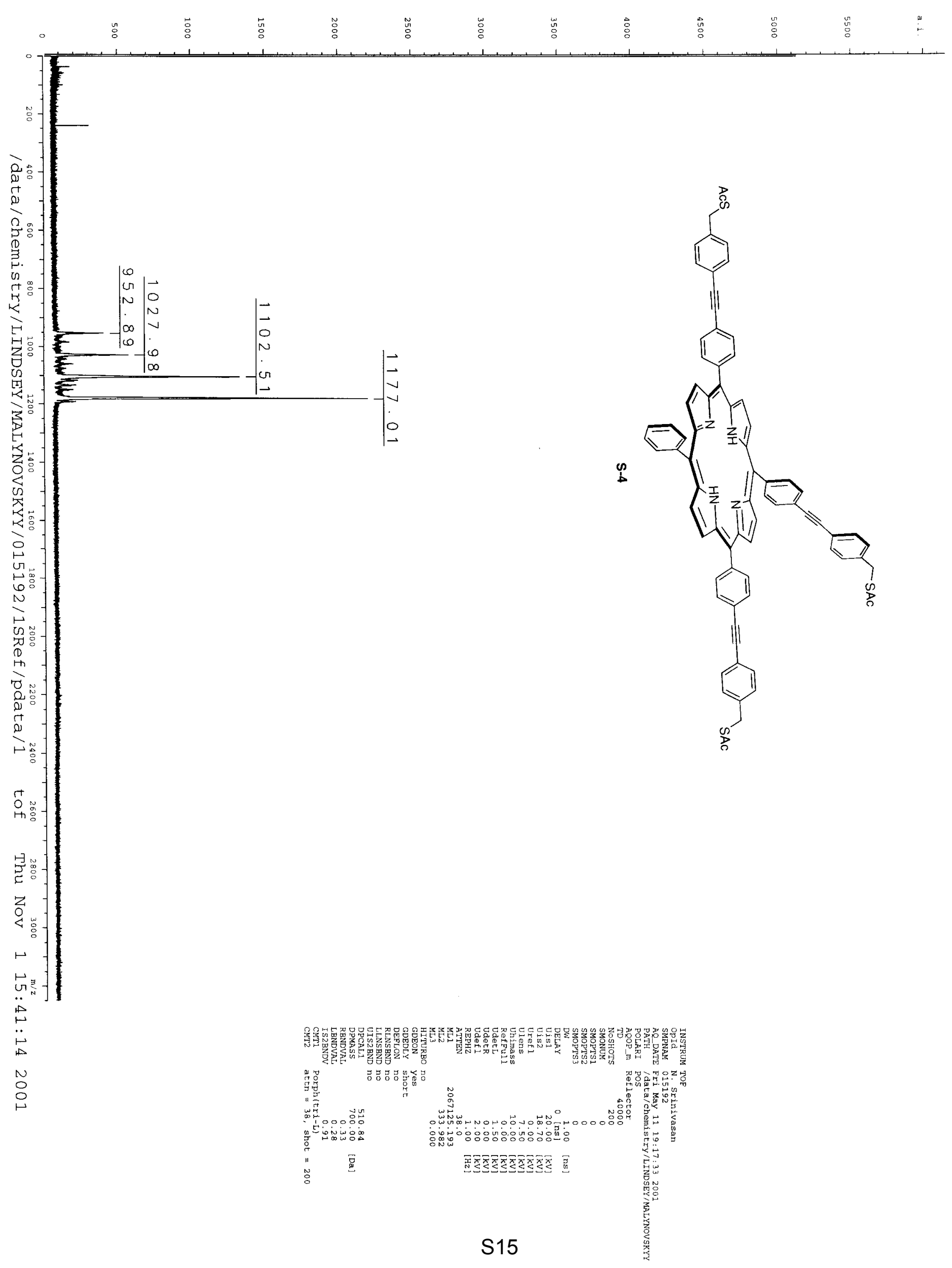




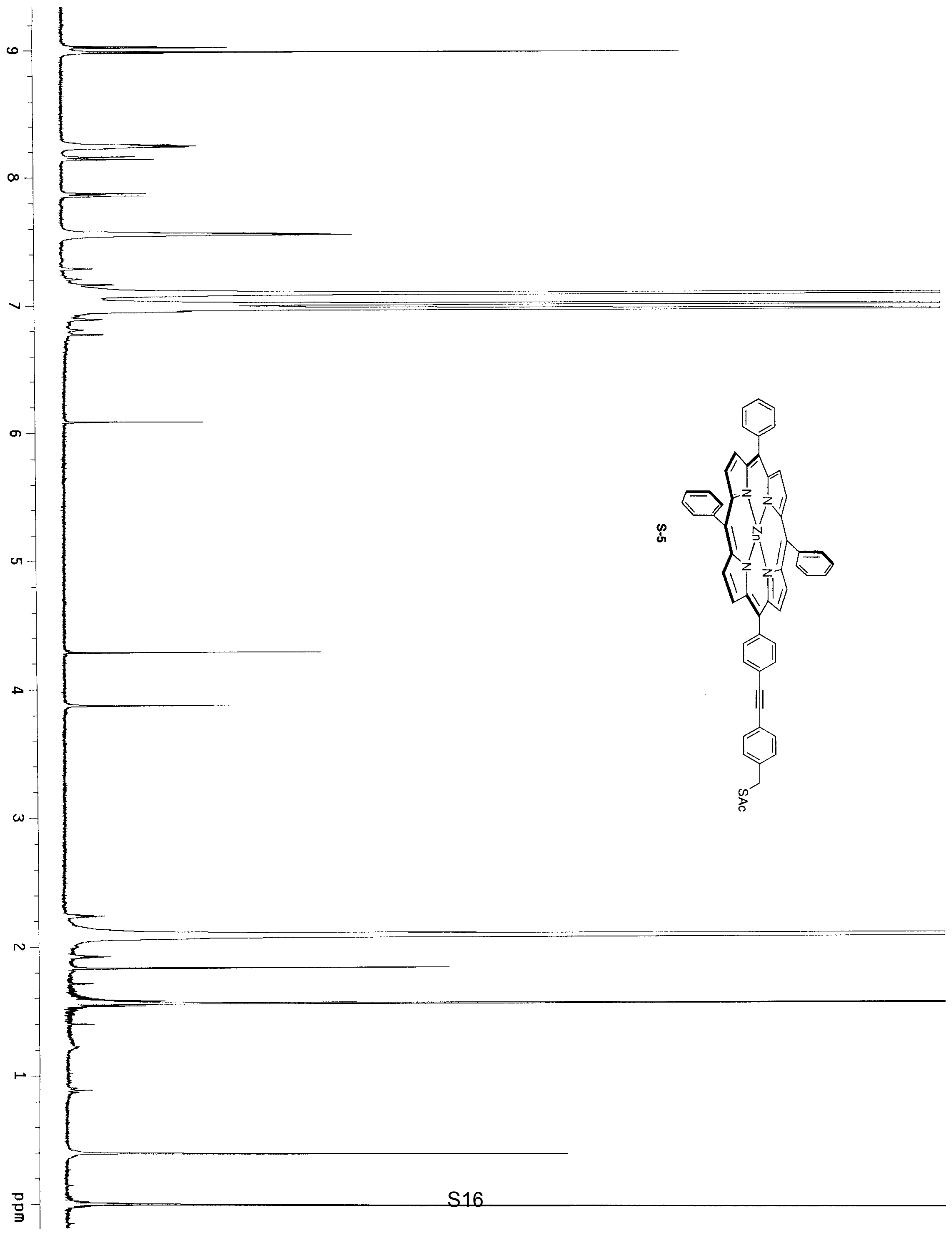




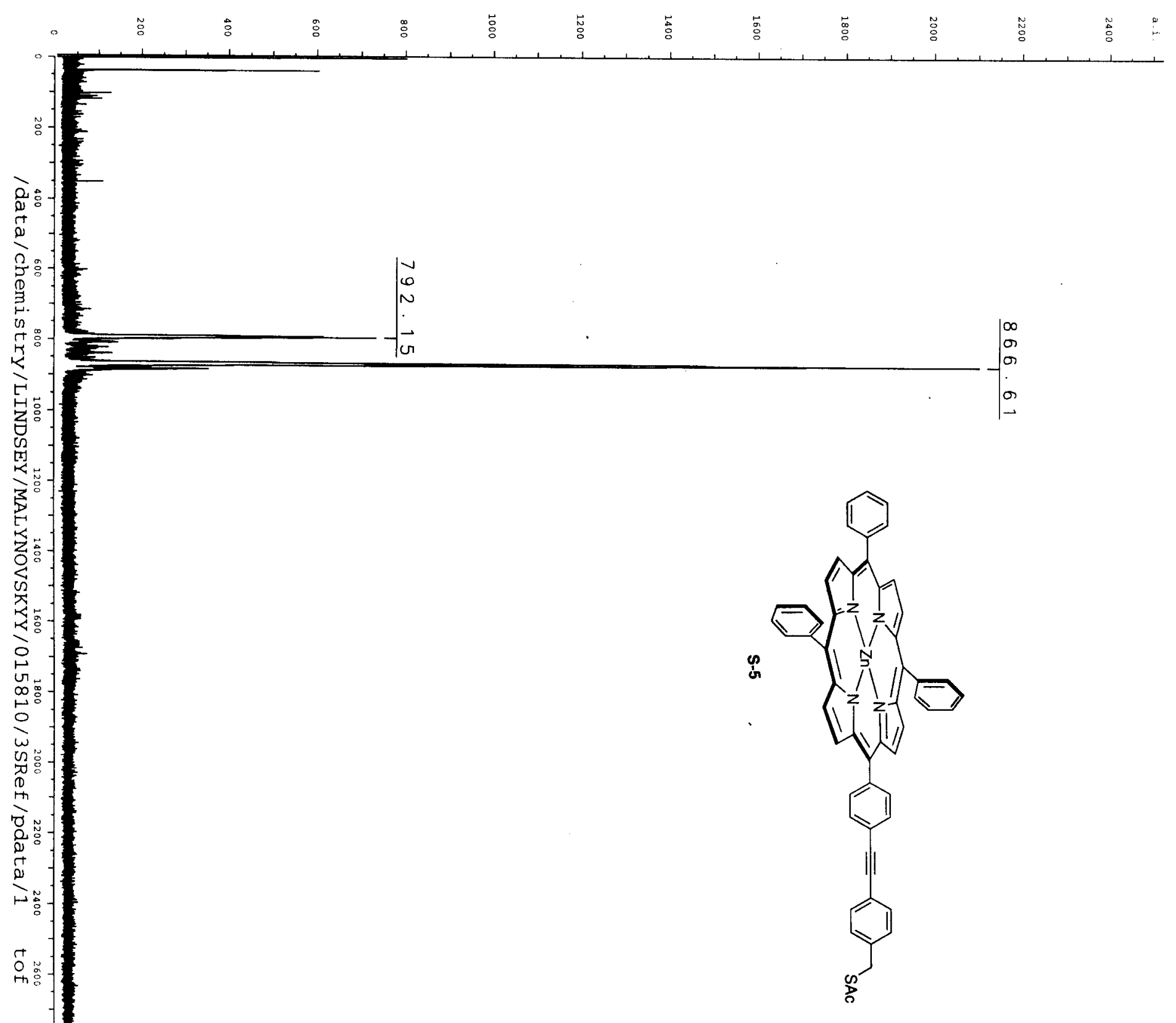

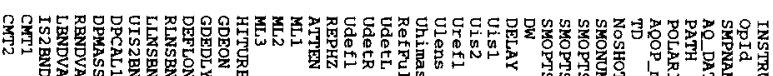

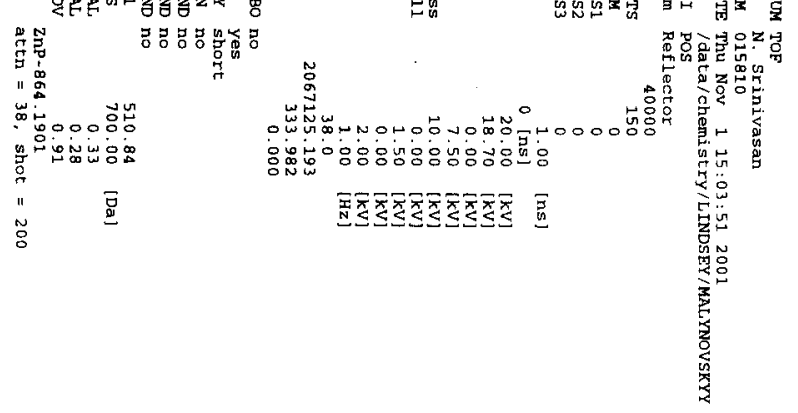




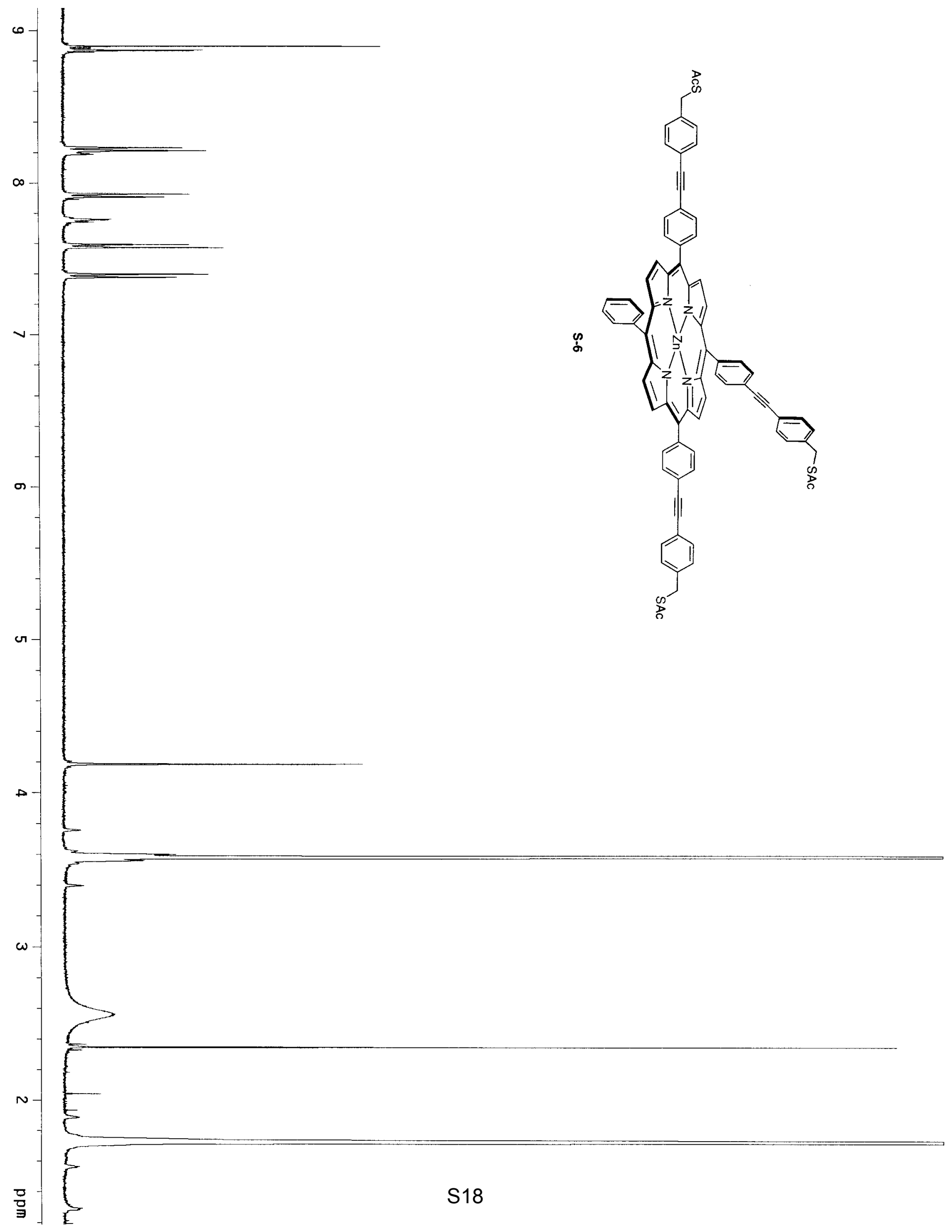




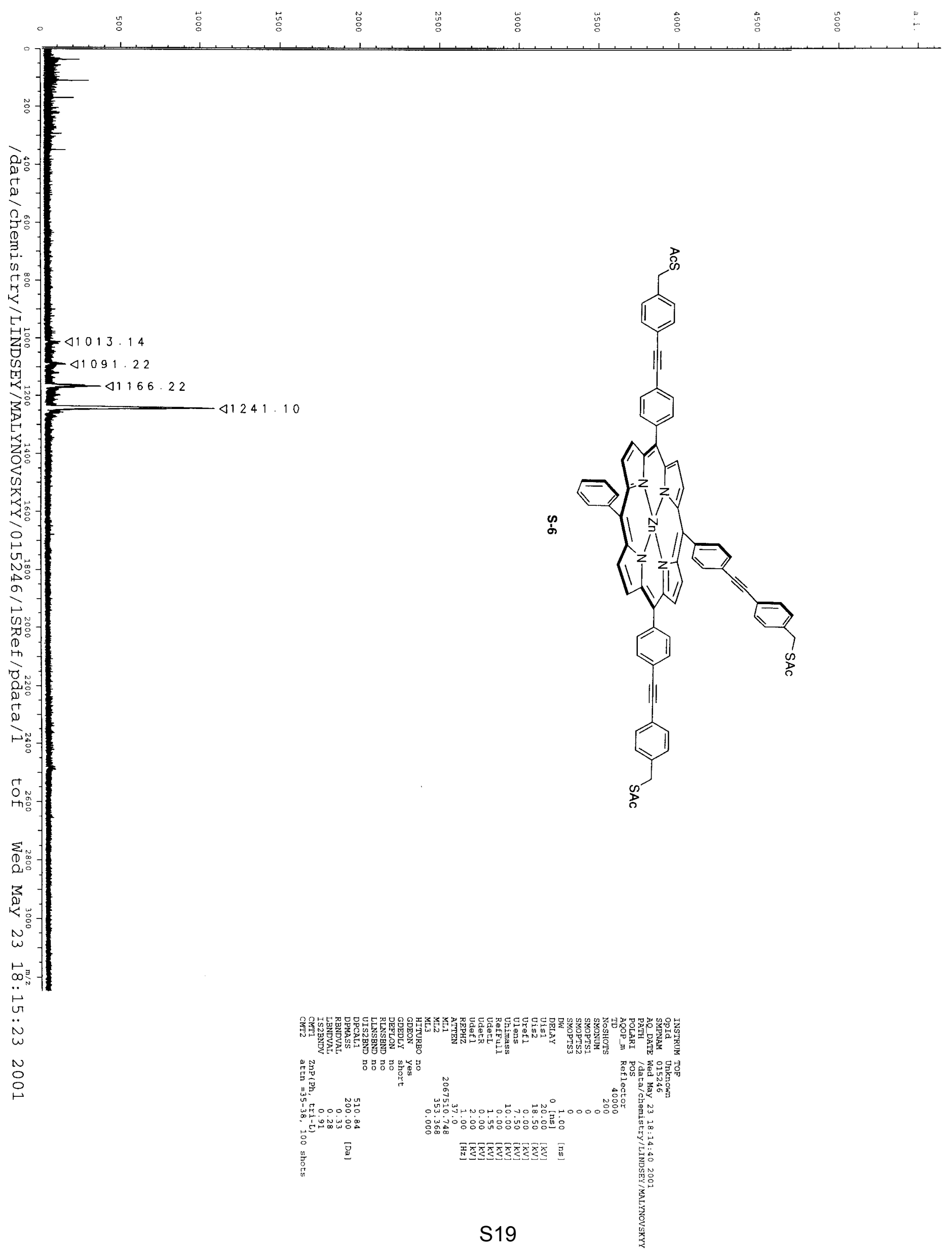




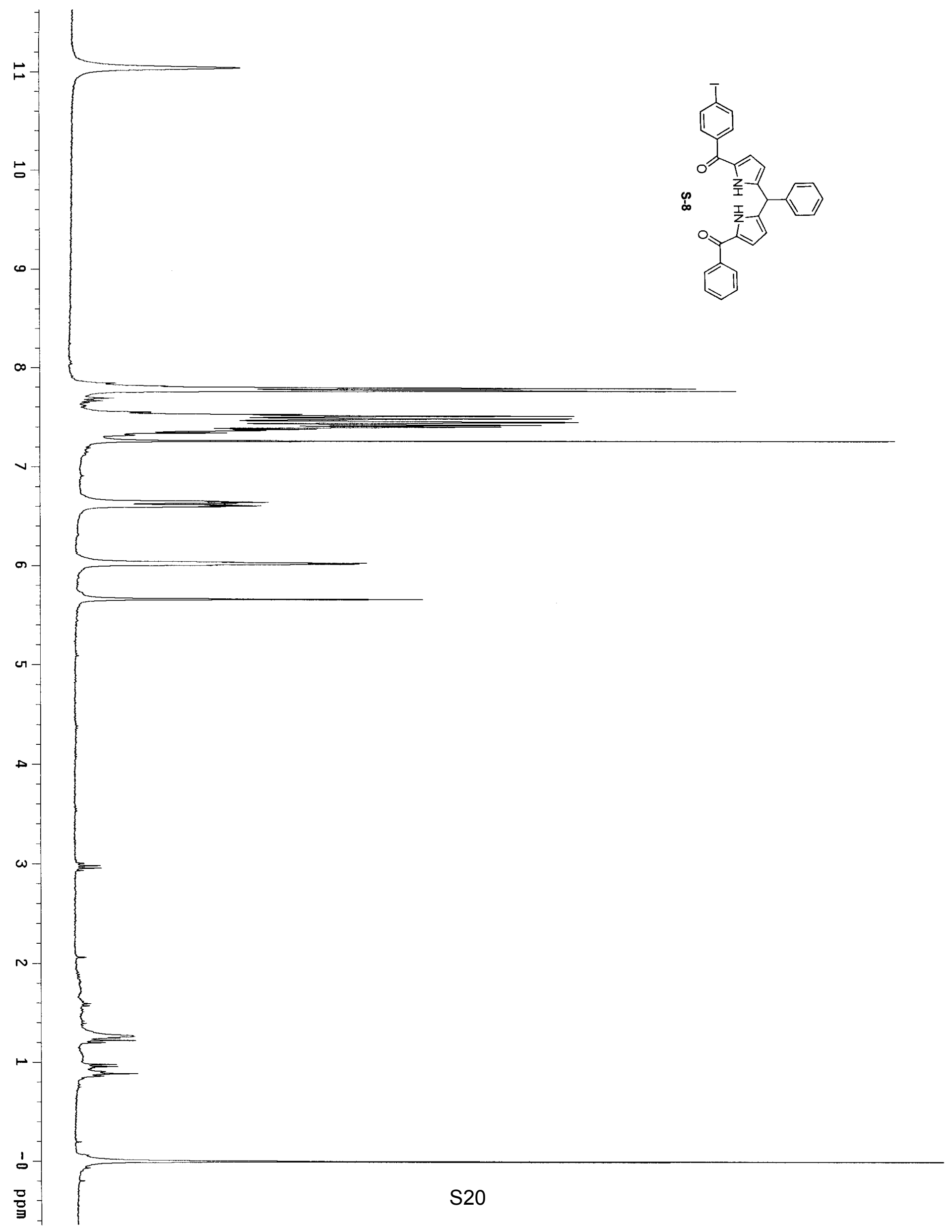




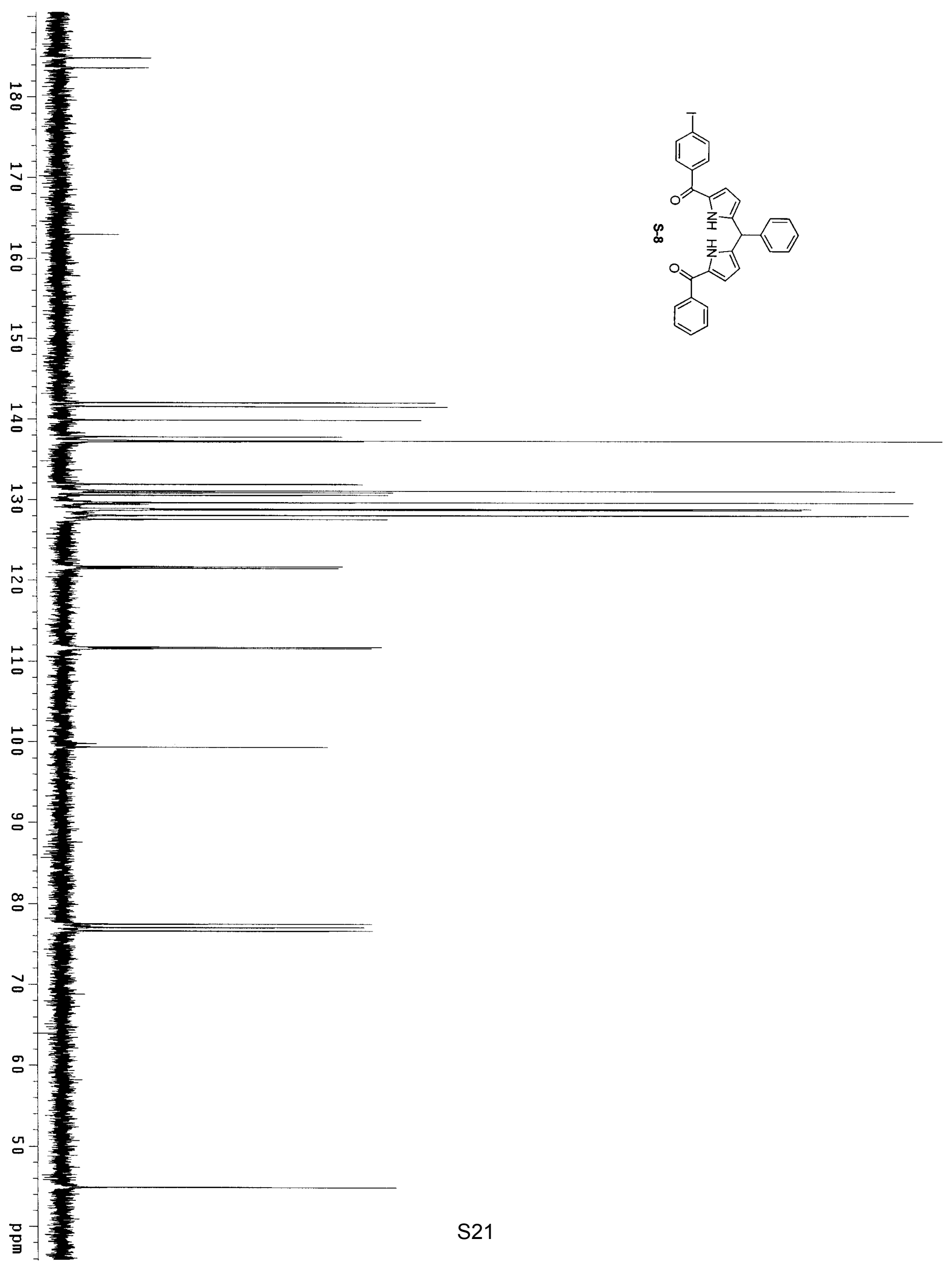




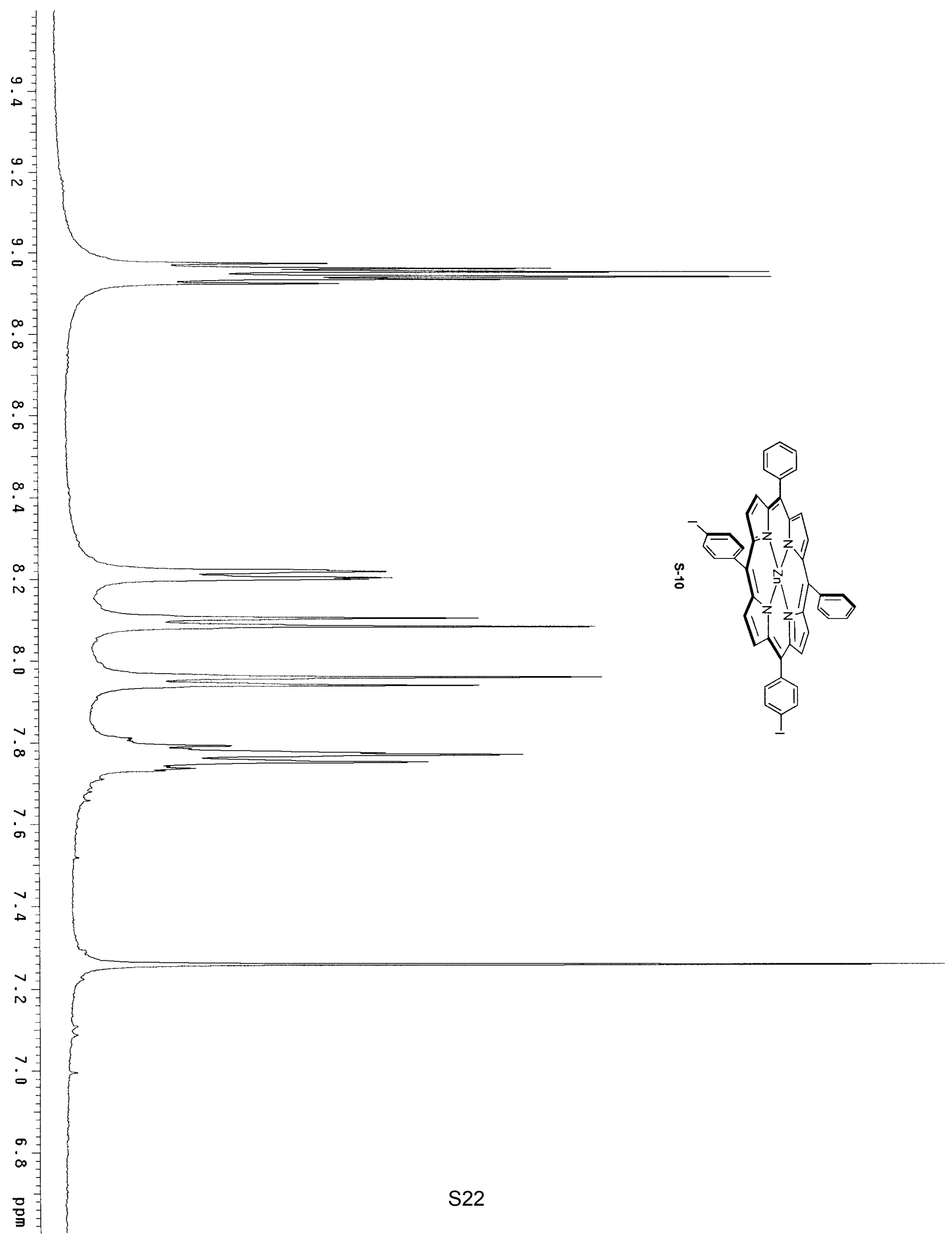



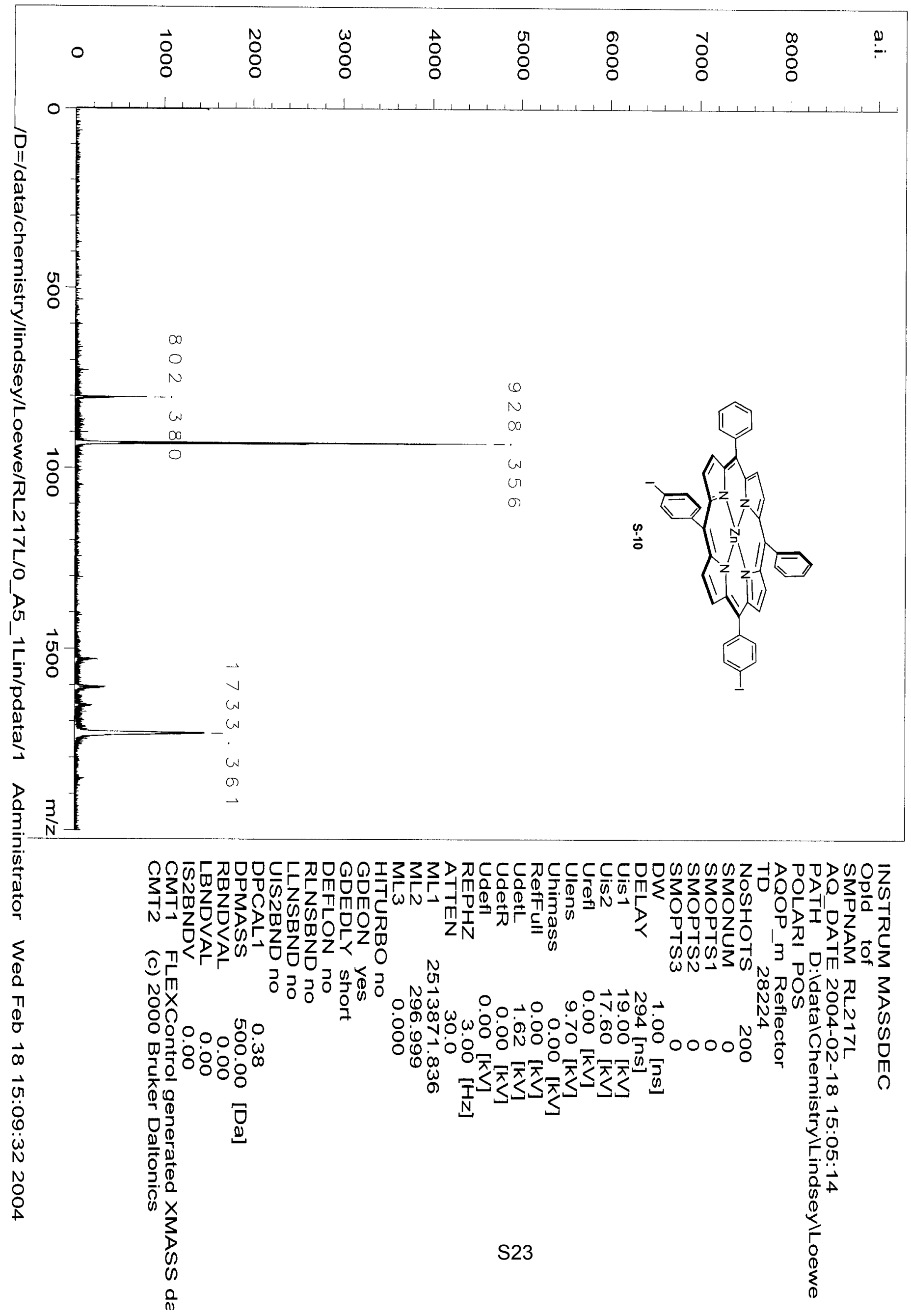


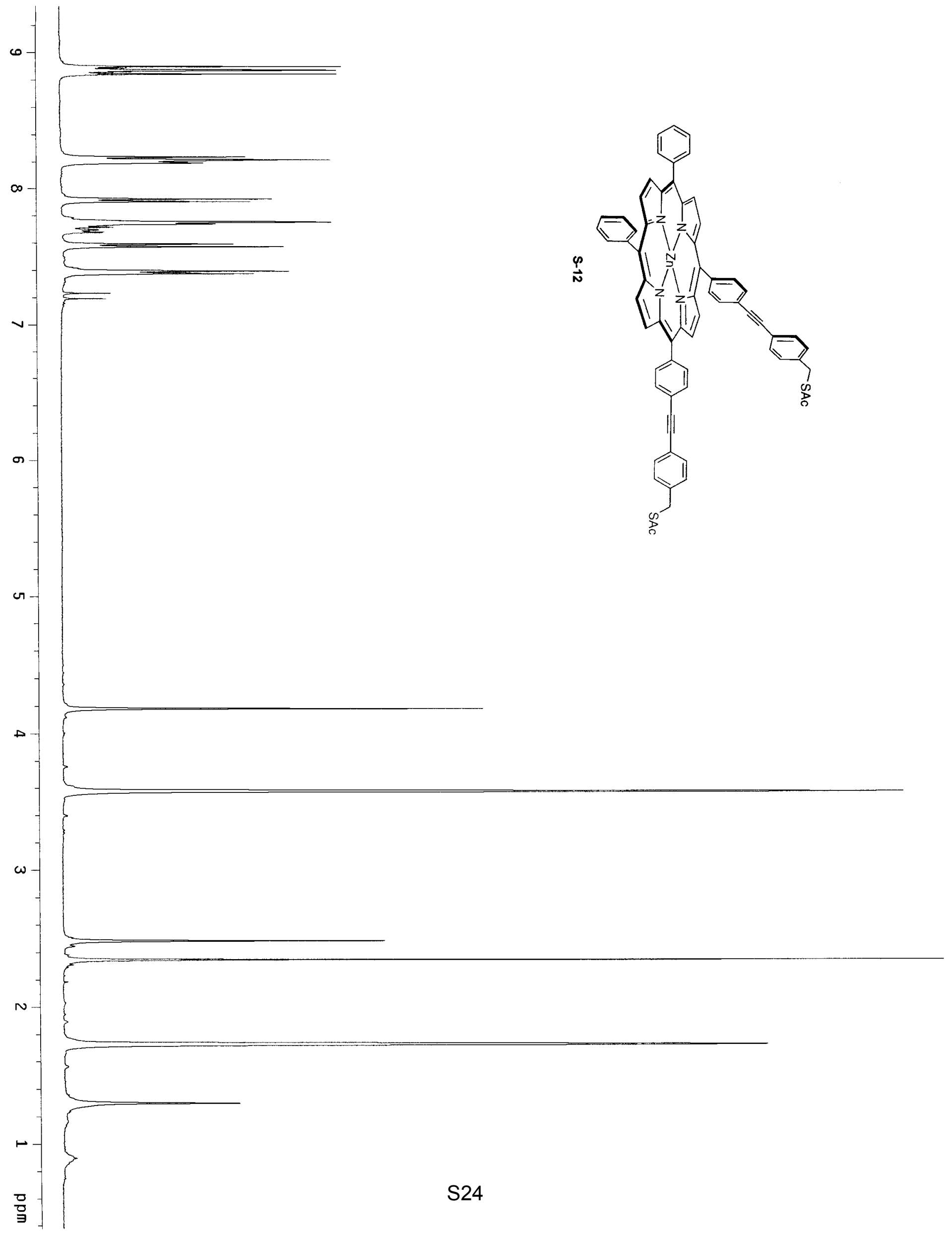




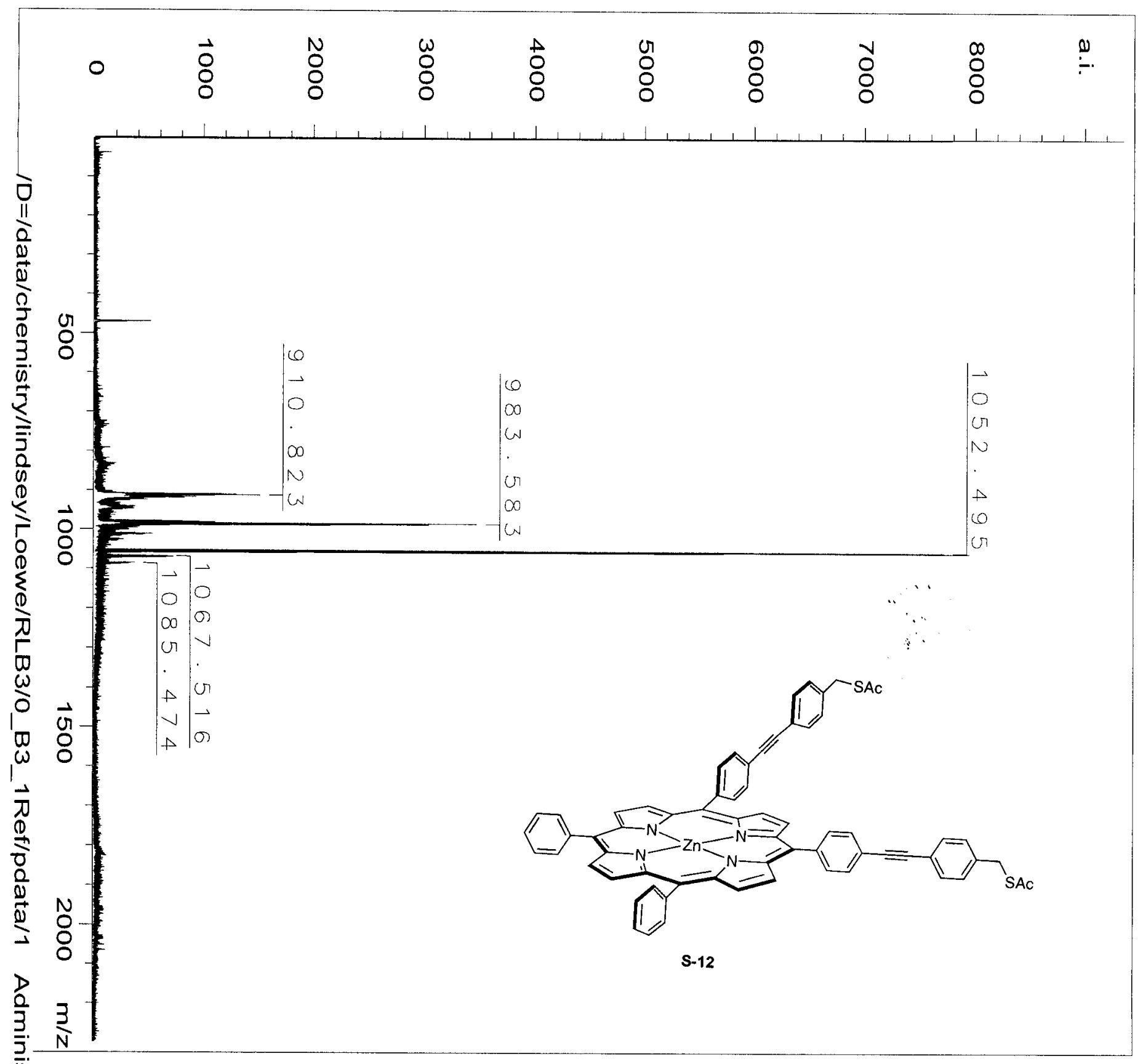

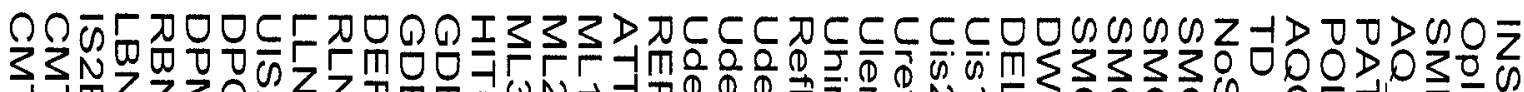

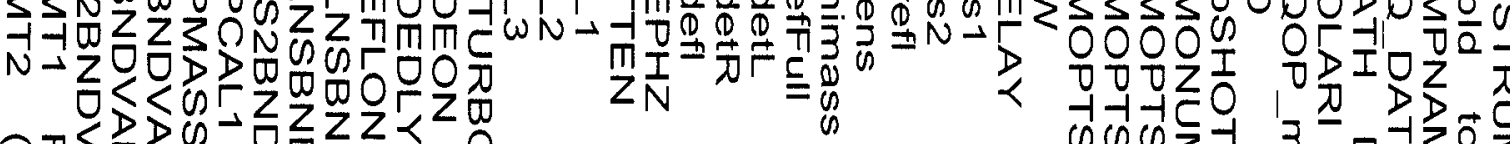

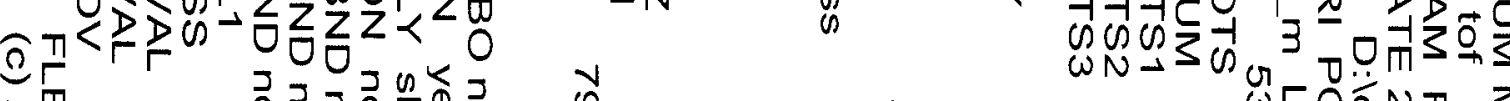

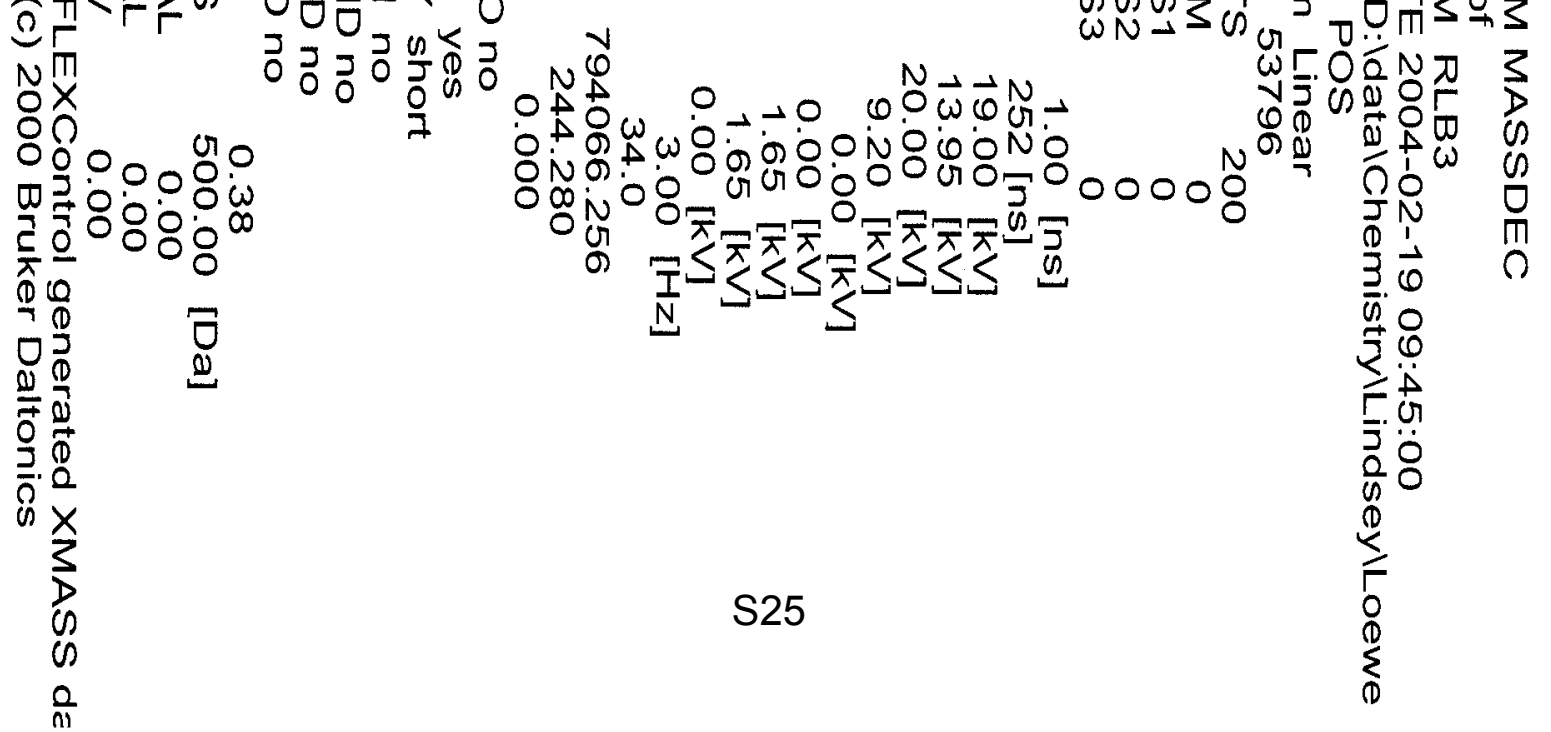




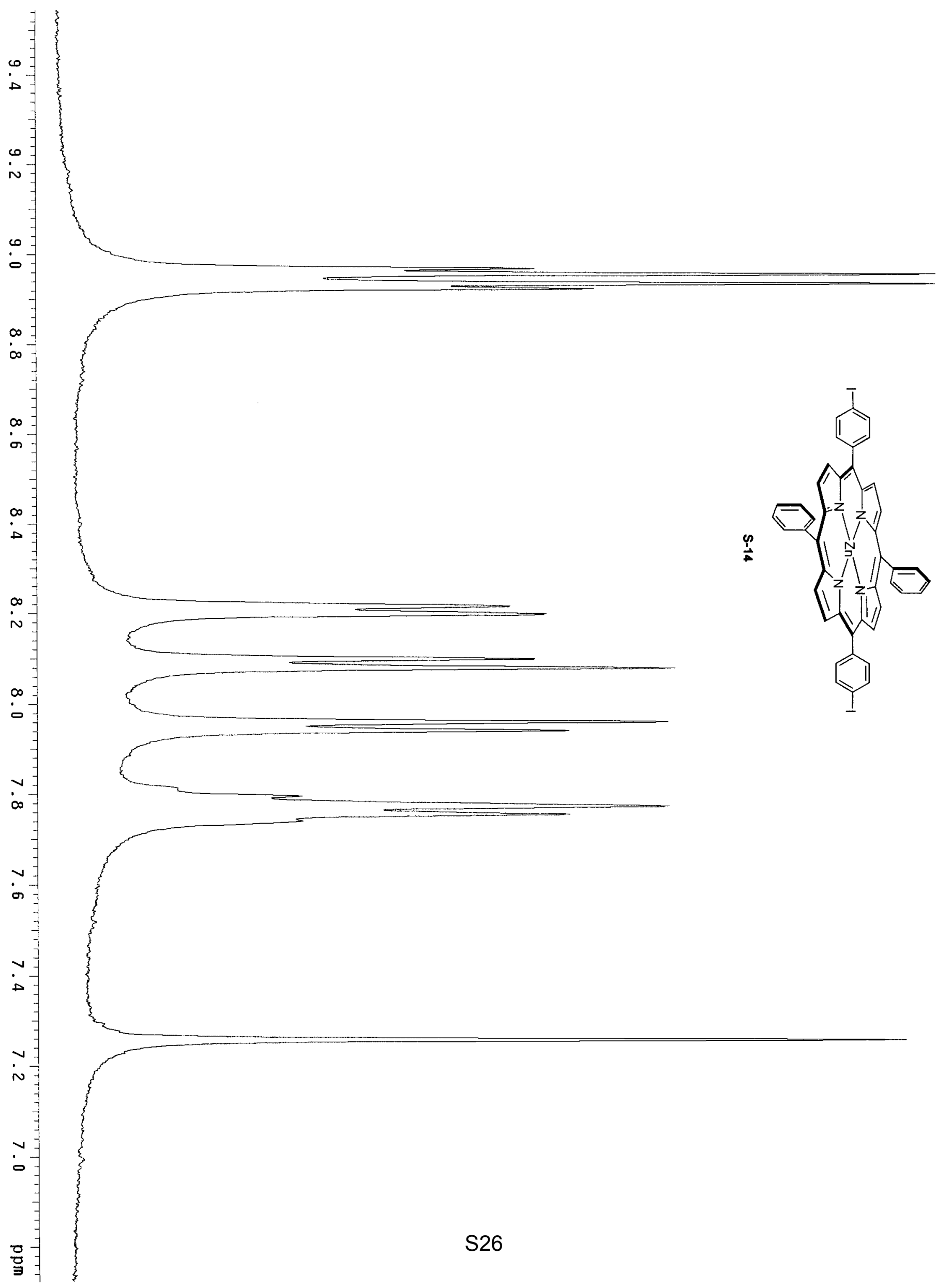




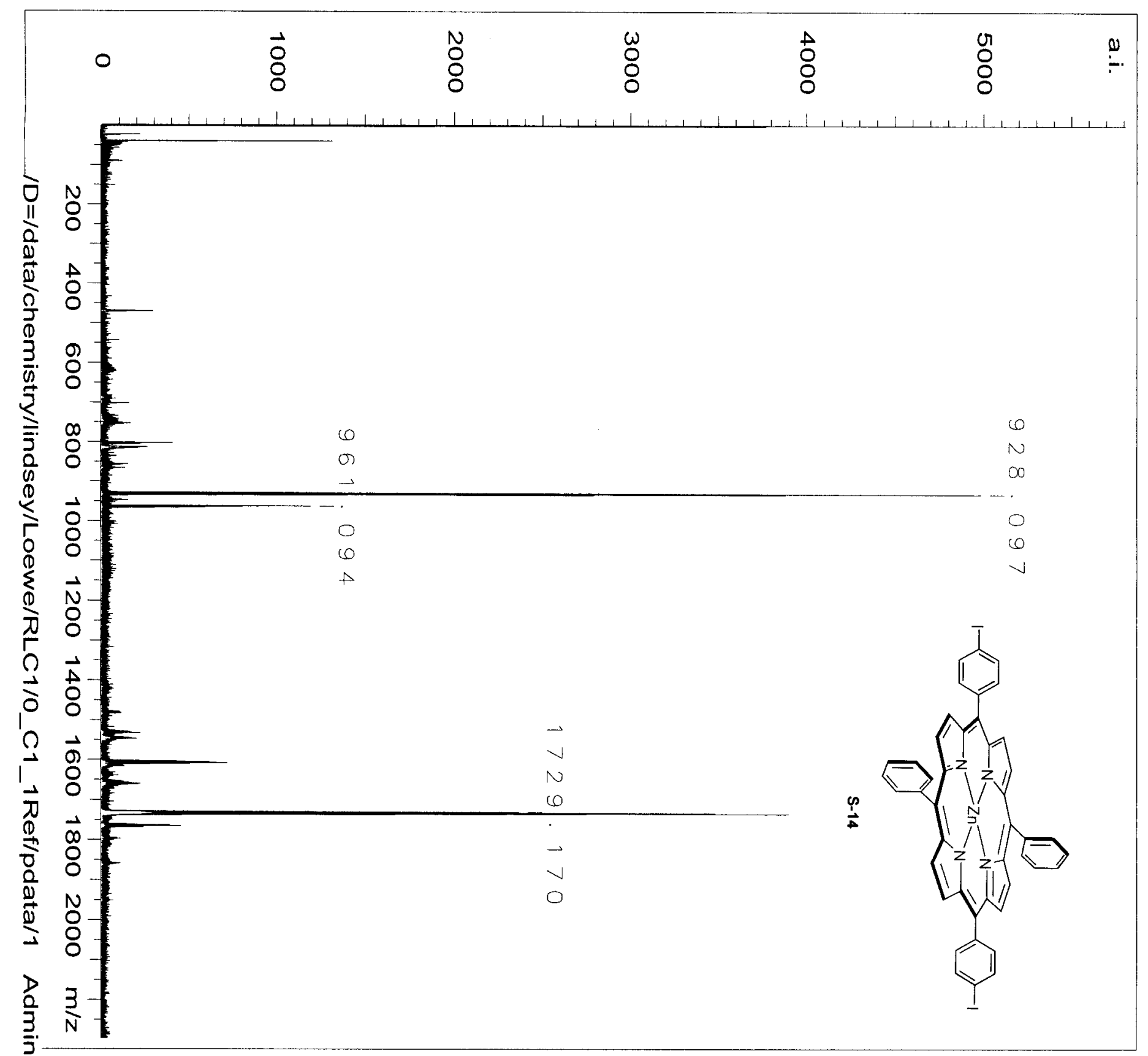

ภดตั

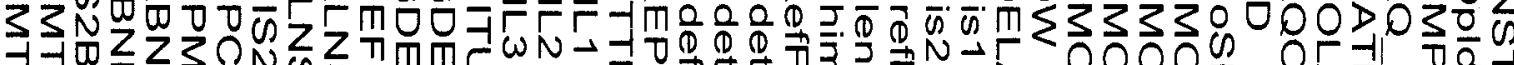

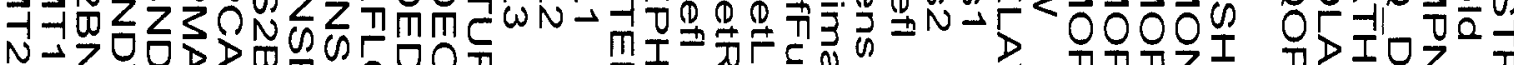

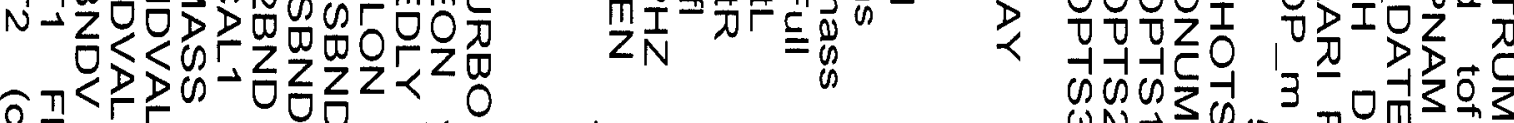

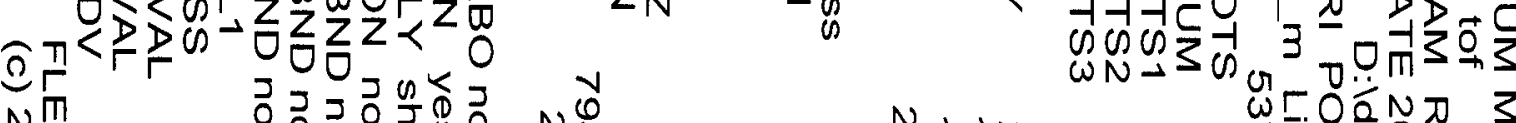

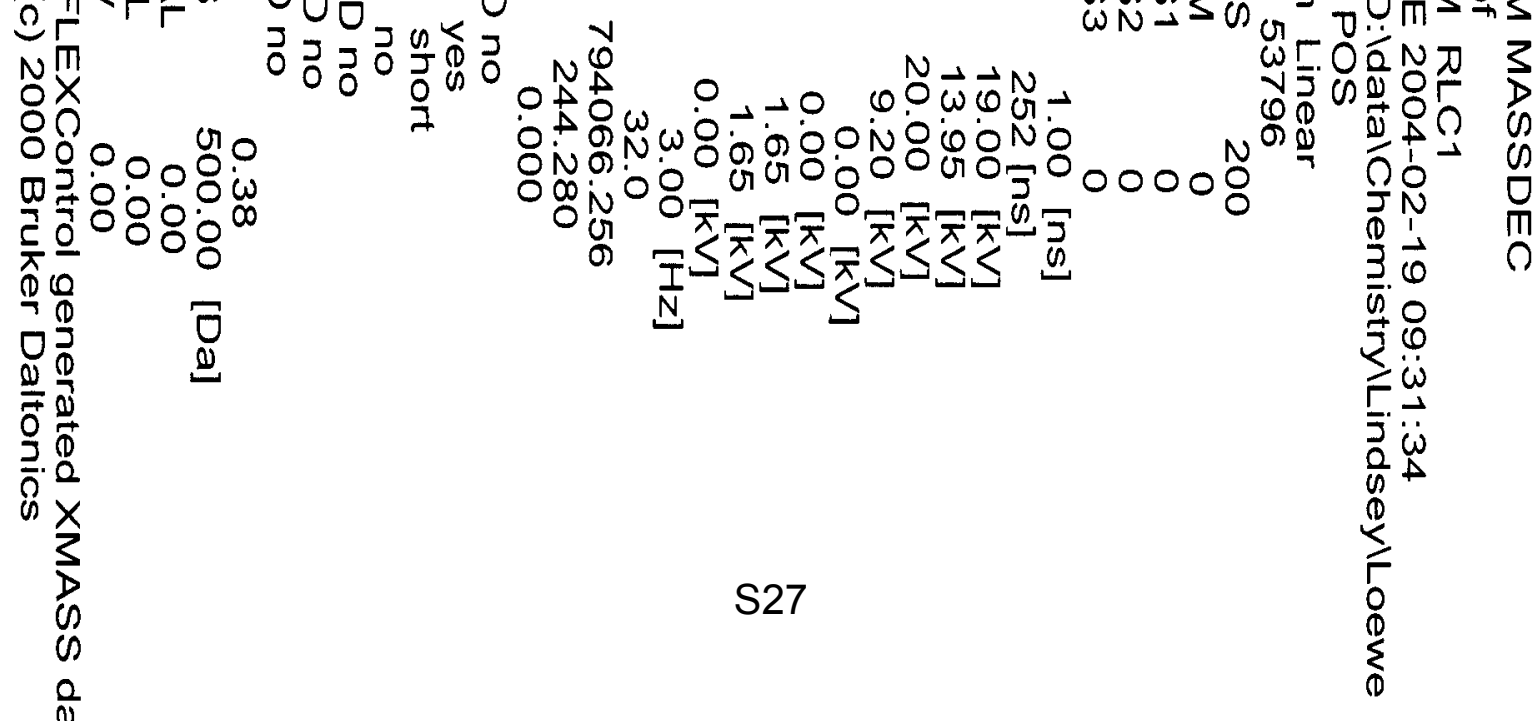




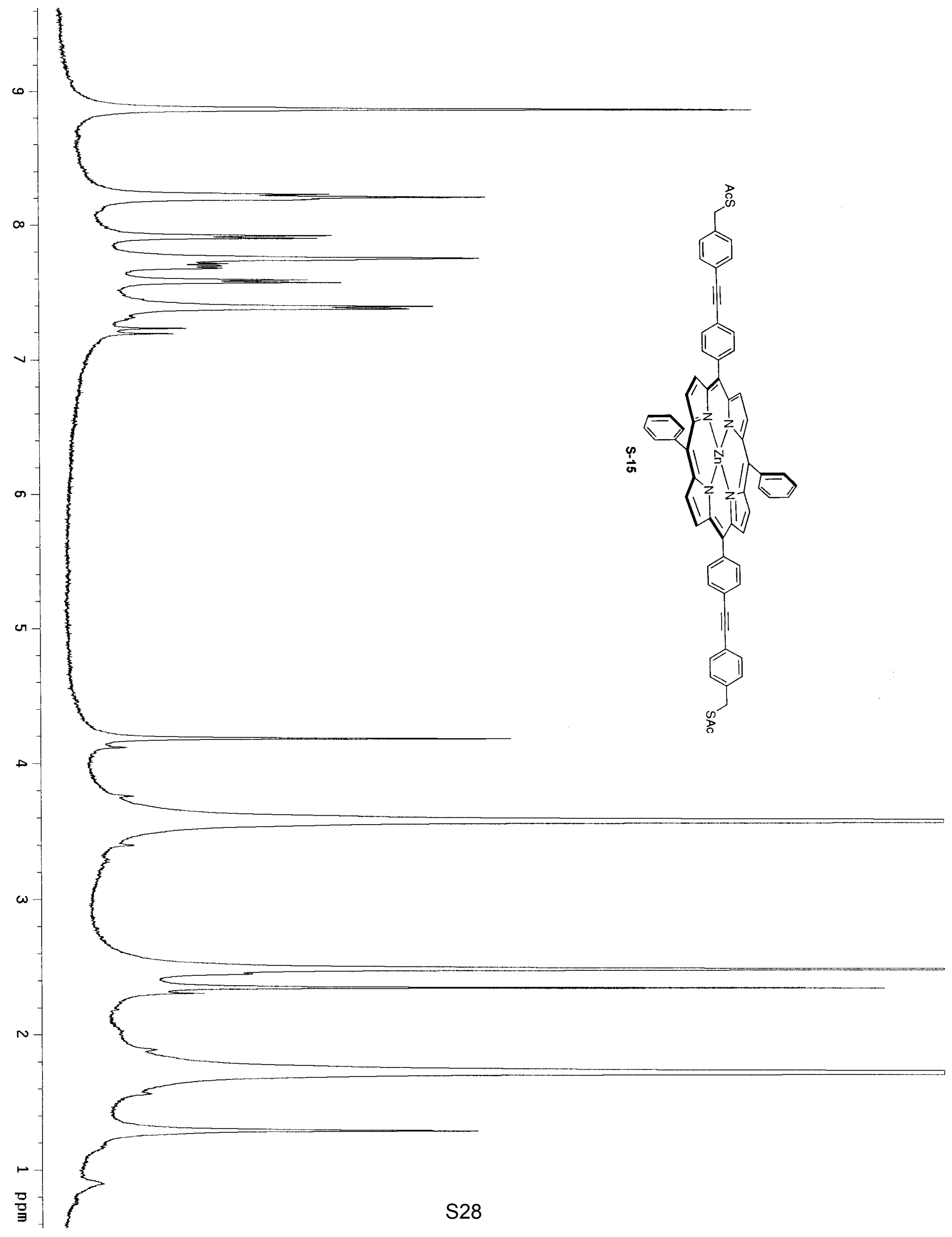




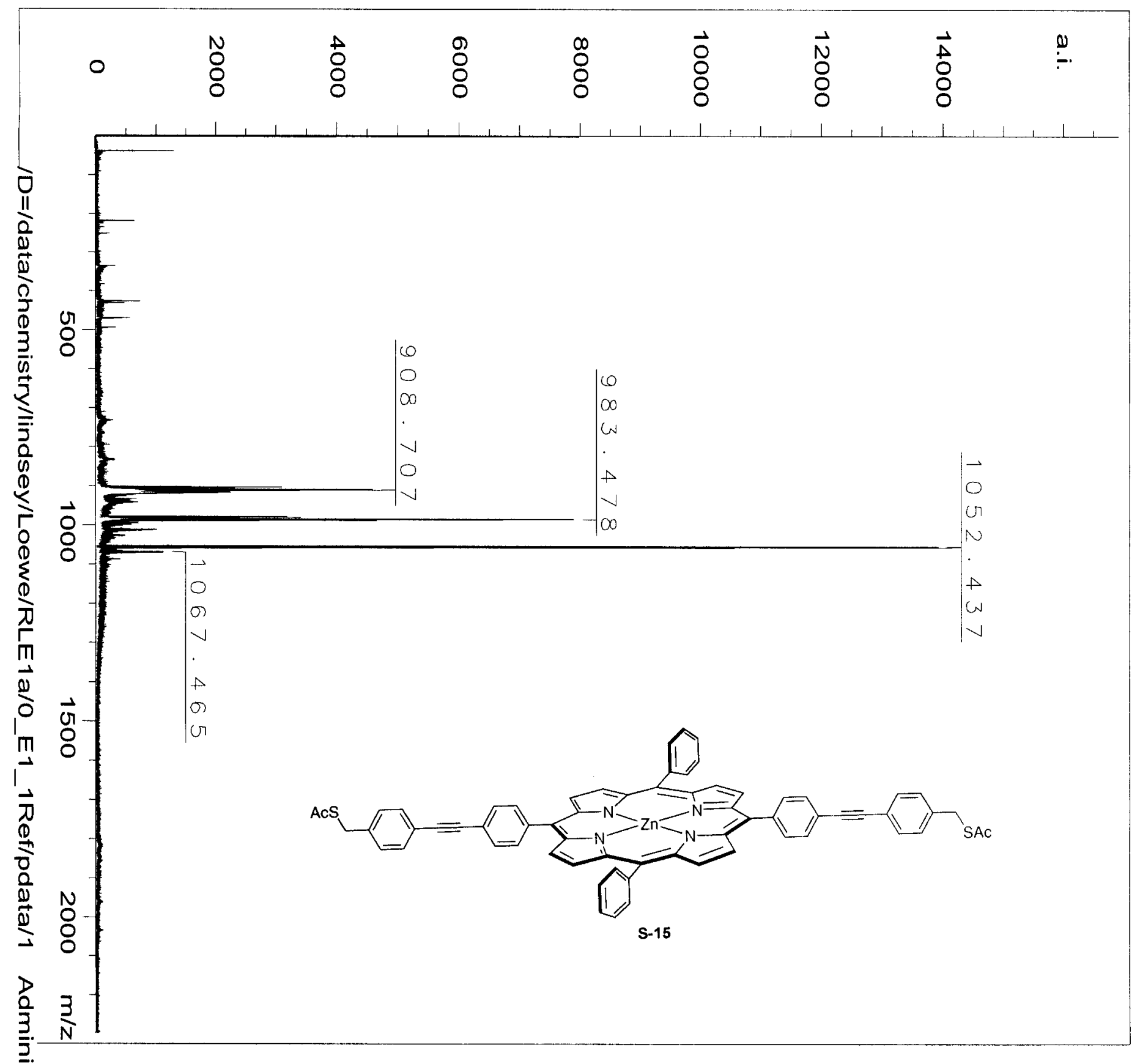

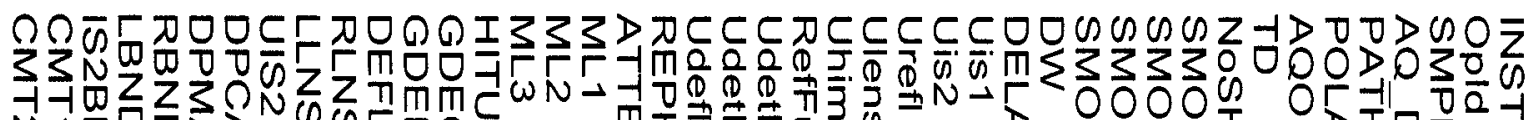

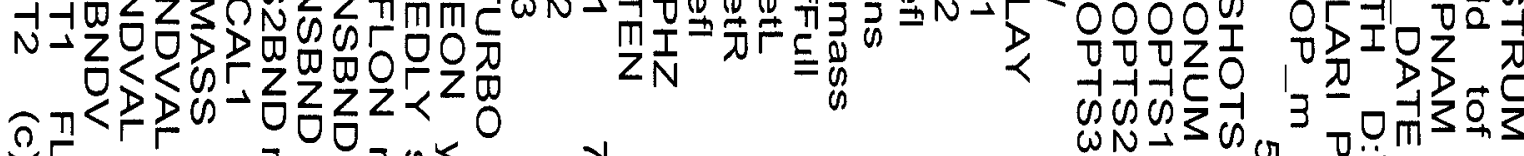

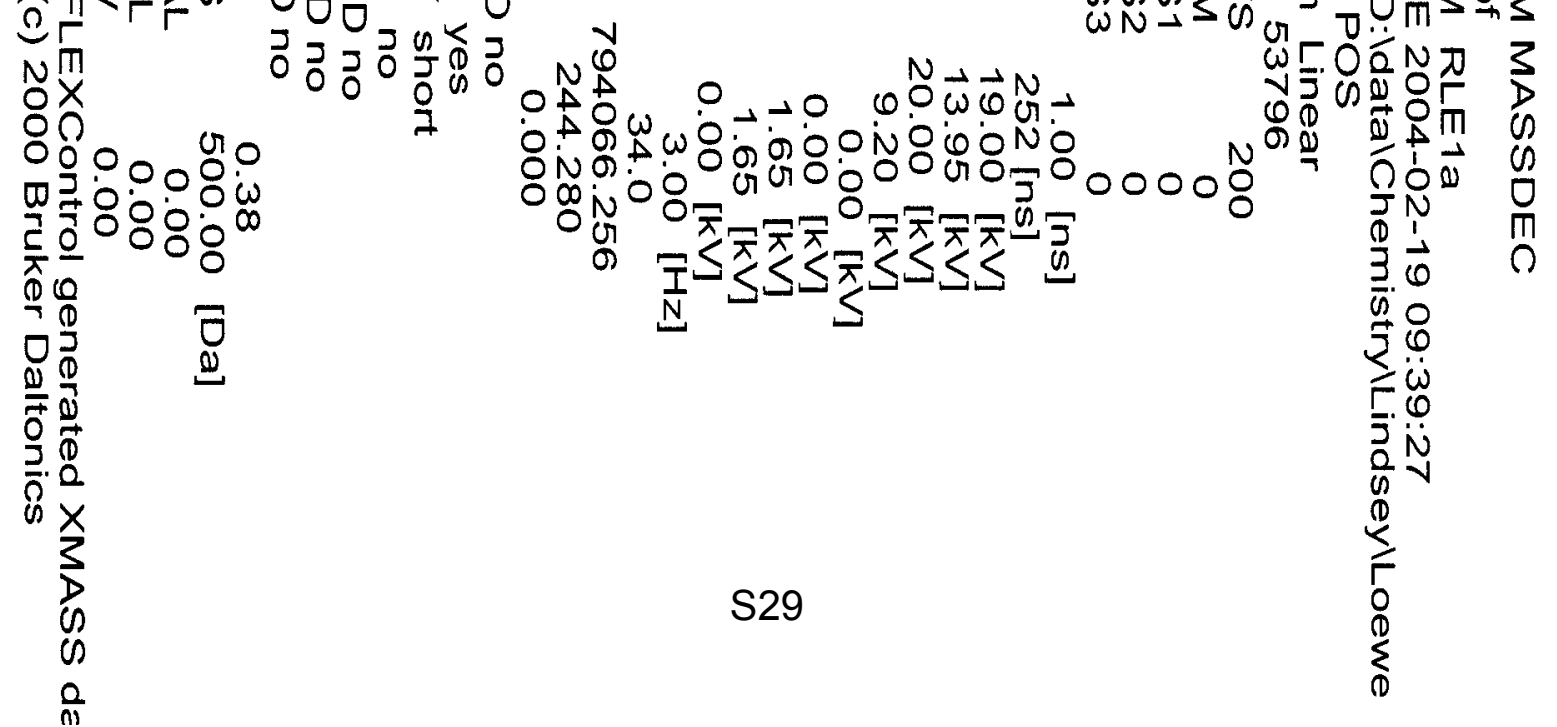

